\title{
Stability of the magnetic Couette-Taylor flow
}

\author{
B. Scarpellini
}

Dedicated to Prof. H. Amann on the occasion of his 65. birthday

\begin{abstract}
In this paper we consider the magnetic Couette-Taylor problem, that is, a conducting fluid between two infinite rotating cylinders, subject to a magnetic field parallel to the rotation axis. This configuration admits an equilibrium solution of the form $\left(0, a r+b r^{-1}, 0,0,0, \alpha+\right.$ $\beta \log r)$. It is shown that this equilibrium is Ljapounov stable under small perturbations in $\mathcal{L}^{2}(\Gamma)$, where $\Gamma=\left\{(r, \varphi, z) / r_{1}<r<r_{2}, \varphi \in[0,2 \pi], z \in \mathbb{R}\right\}$, provided that the parameters $a, b, \alpha, \beta$ are small. The methods of proof are a combination of an energy method, based on Bloch space analysis and small data techniques.
\end{abstract}

Mathematics Subject Classification (2000). 35Q30, 35Q35, 76E07, 76E25.

Keywords. Magnetic Couette-Taylor problem, Ljapounov stability, Bloch space, small data techniques

\section{Introduction}

There are a number of special fluid flows, whose stability properties have attracted attention. Among these there are the Bénard problem (BP), the Couette-Taylor problem (CT), the magnetic Bénard problem (MBP) and the magnetic CouetteTaylor problem (MCT). About BP and CT there is an extended literature. We refer to [7], [11], [3], [4], [9] which treat these problems partly from a theoretical and partly from an applied point of view, and which may serve as a guide to the vast literature. As to MBP we refer to [6], [20], [23] for a discussion of MBP under various aspects. Less seems to be known in case of MCT; in fact, despite some efforts we were not able to find a strictly mathematical discussion of the stability properties related to MCT. We briefly sketch the problem and refer to [2], chpt. IX for more details.

A conducting, viscous, incompressible fluid fills the space between two rotating cylinders with radii $r_{1}<r_{2}$ and angular velocities $\omega_{1}, \omega_{2}$ respectively, thus filling the unbounded domain

$$
\Gamma=\left\{(r, \varphi, z) / r_{1}<r<r_{2}, \varphi \in[0,2 \pi], z \in \mathbb{R}\right\} .
$$


Here, $\Gamma$ is described in terms of cylindrical coordinates to be used throughout what follows. Besides the velocity field $u=\left(u_{1}, u_{2}, u_{3}\right)$ and the pressure $p$ one also has a magnetic field $H=\left(H_{1}, H_{2}, H_{3}\right)$ which interacts with the fluid. The evolution in time of $u, H, p$ is described by a system of equations, referred to as MHD for short, which will be written down in the next section. This system admits an equilibrium solution of the following form:

$$
\begin{aligned}
u_{0} & =(0, V(r), 0), \quad H_{0}=(0,0, \alpha+\beta \log r), r_{1} \leq r \leq r_{2}, \\
v & =V(r)=a r+b r^{-1}, \quad \frac{\partial p_{0}}{\partial r}=V^{2} r^{-1}, \text { any } a, b, \alpha, \beta .
\end{aligned}
$$

If $\beta=0$ then (1.2) is referred to as the trivial solution; the case $\beta \neq 0$ has not found attention, presumably because it is not of physical interest. We nevertheless include it into our considerations. Our aim is to investigate the stability of the equilibrium solution in (1.2). The result to be proved is: $\left(^{*}\right)$ the solution (1.2) is Ljapounov stable under small $\mathcal{L}^{2}(\Gamma)$-perturbations, provided $a, b, \alpha, \beta$ are small. In [2], the stability of (1.2) for $\beta=0$ is investigated under the following assumptions: (1) the allowed perturbations are radially symmetric and $L$-periodic in the $z$ direction for some fixed $L,(2)$ a so called small gap condition is imposed, (3) the stability analysis is linear.

We comment briefly on the methods used in the proof of $\left(^{*}\right)$. The spectrum of the linearisation of MHD around $u_{0}, H_{0}$ is continuous, in the left halfplane but with points on the imaginary axis (for $a, b, \alpha, \beta$ small), ie. we have marginal stability. This causes us to proceed as in [16] in case of the MBP and to treat the stability problem as a problem of small initial data. These arguments in [16] entail Ljapounov stability of $\left(u_{0}, H_{0}\right)$ in $(1.2)$ provided that the perturbations $\left(v_{0}, h_{0}\right)$ of $\left(u_{0}, H_{0}\right)$ induce solutions $(v(t), h(t))$ of the nonlinear perturbation equations which satisfy a weak energy inequality, ie.:

$$
\partial_{t}\left(\|v(t)\|^{2}+\|h(t)\|^{2}\right) \leq 0
$$

The proof of (1.3), while easy in case of the MBP ([6],[15]), is now considerably more difficult and absorbs the major part of the paper. The nonlinear part of the proof however is only outlined since it is based on the same arguments as those given in [16].

\section{The equations of MHD}

The system MHD is given in [2], pg. 383 in terms of cylindrical coordinates. Since it will be restructured in terms of various operators we reproduce it in detail. That is we write down the perturbation equations around the equilibrium solution in (1.2). The system is in dimensional form but by scaling we have achieved that the factor of $(H \nabla) H$ in [2] is now $=1$. Based on (1.2) we set

$$
\phi=\phi(r)=\alpha+\beta \log r, \quad \Omega(r)=V(r) r^{-1}=V r^{-1} .
$$


We replace $u, H, p$ in [2], pg. 383 by $u_{0}+v, H_{0}+h, p_{0}+p$ where $v=\left(v_{r}, v_{\varphi}, v_{z}\right)$, $h=\left(h_{r}, h_{\varphi}, h_{z}\right)$ and use that $u_{0}, H_{0}, p_{0}$ is an equilibrium solution; we then get

$$
\begin{aligned}
\partial_{t} v_{r}= & \mathcal{V}\left(\Delta v_{r}-\frac{v_{r}}{r^{2}}-\frac{2}{r^{2}} \partial_{\varphi} v_{\varphi}\right)+\phi \partial_{z} h_{r}-\Omega \partial_{\varphi} v_{r}+2 \Omega v_{\varphi} \\
+ & \partial p+(h \nabla) h_{r}-(v \nabla) v_{r}+\frac{v_{\varphi}^{2}}{r}-\frac{h_{\varphi}^{2}}{r} \\
\partial_{t} v_{\varphi}= & v\left(\Delta v_{\varphi}-\frac{v_{\varphi}}{r^{2}}+\frac{2}{r^{2}} \partial_{\varphi} v_{r}\right)+\phi \partial_{z} h_{\varphi}-\Omega \partial_{\varphi} v_{\varphi}-\left(\frac{V}{r}+\partial V\right) v_{r} \\
+ & \frac{1}{r} \partial_{\varphi} p+(h \nabla) h_{\varphi}-(v \nabla) v_{\varphi}+\frac{h_{\varphi} h_{r}}{r}-\frac{v_{\varphi} v_{r}}{r} \\
\partial_{t} v_{z}= & \mathcal{V} \Delta v_{z}+\partial_{z} p+\phi \partial_{z} h_{z}+h_{r} \partial \phi-\Omega \partial_{\varphi} v_{z} \\
+ & (h \nabla) h_{z}-(v \nabla) v_{z} \\
\partial_{t} h_{r}= & \eta\left(\Delta h_{r}-\frac{h_{r}}{r^{2}}-\frac{2}{r^{2}} \partial_{\varphi} h_{\varphi}\right)+\phi \partial_{z} v_{r}-\Omega \partial_{\varphi} h_{r}+(h \nabla) v_{r}-(v \nabla) h_{r} \\
\partial_{t} h_{\varphi}= & \eta\left(\Delta h_{\varphi}-\frac{h_{\varphi}}{r^{2}}+\frac{2}{r^{2}} \partial_{\varphi} h_{r}\right)+h_{r} \partial V+\phi \partial_{z} v_{\varphi}-\Omega \partial_{\varphi} h_{\varphi}-\Omega h_{r} \\
+ & (h \nabla) v_{\varphi}-(v \nabla) h_{\varphi}-\frac{1}{r}\left(v_{\varphi} h_{r}-h_{\varphi} v_{r}\right) \\
\partial_{t} h_{z}= & \eta \Delta h_{z}+\phi \partial_{z} v_{z}-\Omega \partial_{\varphi} h_{z}-v_{r} \partial \phi+(h \nabla) v_{z}-(v \nabla) h_{z} \\
& \frac{1}{2} \partial r h_{r}+\frac{1}{r} \partial_{\varphi} h_{\varphi}+\partial_{z} h_{z}=0, \quad \frac{1}{r} \partial r v_{r}+\frac{1}{r} \partial_{\varphi} v_{\varphi}+\partial_{z} v_{z}=0
\end{aligned}
$$

Here, $\partial, \partial_{\varphi}, \partial_{z}$ are the derivatives with respect to $r, \varphi, z$ and

$$
(h \nabla) v=h_{r} \partial v_{r}+\frac{h_{\varphi}}{r} \partial_{\varphi} v_{\varphi}+h_{z} \partial_{z} v_{z} ;
$$

likewise in the other cases. The Laplacian $\Delta$ is in cylindrical coordinates; $\mathcal{V}$ is the viscosity, $\eta$ the electrical conductivity. The boundary conditions depend on the electrical properties of the cylinders; as in [2] we assume that the walls are fully conducting. With the flow subject to nonslip boundary conditions we are led to require (see [2], IV, IX):

$$
v=0, \quad, \vec{n} \cdot h=0 \quad \text { and } \quad \operatorname{rot} h \times n=0 \text { at } \partial \Gamma,
$$

where $\vec{n}$ is the outer unit normal at a generic point on $\partial \Omega$. In terms of cylinder coordinates, (2.2) assumes the form:

$$
v=0, \quad h_{r}=0, \quad \partial\left(r h_{\varphi}\right)=0, \quad \partial h_{z}=0 \text { at } \partial \Gamma,
$$

that is $v_{r}, v_{\varphi}, v_{z}, h_{r}$ satisfy Dirichlet conditions, $h_{z}$ Neumann conditions and $h_{\varphi}$ a kind of Robin condition at $\partial \Gamma$. Next we have to impose on the above system a suitable functional frame in which the stability question can be posed properly.

\section{A functional setting}

In order to cast the system MHD in sect. 2 into a suitable functional frame we introduce the usual Sobolev spaces $H^{p}(\Gamma), H_{0}^{p}(\Gamma)$ with $\Gamma$ as in $(1.1) . H^{p}(\Gamma)$ is endowed with the scalar product $(,)_{p}, p \geq 0$; we set $H^{0}(\Gamma)=\mathcal{L}^{2}(\Gamma)$. The product spaces $H^{p}(\Gamma)^{n}$ are endowed with the scalar product $\langle,\rangle_{p}$ where

$$
\langle u, v\rangle_{p}=\sum\left(u_{j} ; v_{j}\right)_{p}, \quad u=\left(u_{1}, \ldots, u_{n}\right), \quad v=\left(v_{1}, \ldots, v_{n}\right) \in H^{p}(\Gamma)^{n} .
$$


The scalar product $\langle,\rangle_{p},(,)_{p}$ induce norms which independently of $n$ are denoted by \|\|$_{H^{p}}$ for $p>0$ and by \|\|$_{\mathcal{L}^{2}}$ or even \|\| for $p=0$; that is we interpret \|\|$_{H^{p}}$, \|\| according to the context. Sobolev norms may be expressed in cartesian or in cylinder coordinates, according to the case. If eg. $u=\left(u_{1}, \ldots, u_{n}\right) \in H^{1}(\Gamma)^{n}$ then $\|u\|_{H^{1}}^{2}=\|u\|^{2}+\|\nabla u\|^{2}$ is given by

$$
\sum_{j} \int_{\Gamma}\left|u_{j}\right|^{2} r d r d \varphi d z+\sum_{j} \int_{\Gamma}\left(\left|\partial u_{j}\right|^{2}+\frac{1}{r^{2}}\left|\partial_{\varphi} u_{j}\right|^{2}+\left|\partial_{z} u_{j}\right|^{2}\right) r d r d \varphi d z
$$

and likewise in other cases. Due to the boundary conditions (2.3) we need two further Sobolev spaces. Recalling $\partial=\partial_{r}$ we set

$$
\begin{aligned}
& f \in \widetilde{H}^{2}(\Gamma) \text { iff } f \in H^{2}(\Gamma) \text { and } \partial f \in H_{0}^{1}(\Gamma), \\
& f \in \widehat{H}^{2}(\Gamma) \text { iff } f \in H^{2}(\Gamma) \text { and } \partial(r f) \in H_{0}^{1}(\Gamma) .
\end{aligned}
$$

Finally, given operators $A_{j}$ on Hilbert spaces $H_{j}$ with domains $D_{j}$ for $j=1, \ldots, n$ then $A=\operatorname{diag}\left(A_{1} \times \cdots \times A_{n}\right)$ is the operator on $H_{1} \times \cdots \times H_{n}$ such that

$$
\begin{aligned}
& \operatorname{dom}(A)=D_{1} \times \cdots \times D_{n} ; \quad A x=\left(A_{1} x_{1}, \ldots, A_{n} x_{n}\right) \\
& \text { for } x=\left(x_{1}, \ldots, x_{n}\right) \text { in } \operatorname{dom}(A) .
\end{aligned}
$$

Next let $\mathcal{V}, \widetilde{\mathcal{V}}$ be given according to

$$
\begin{aligned}
u & =\left(u_{1}, u_{2}, u_{3}\right) \in \widetilde{\mathcal{V}} \text { iff } u_{1} \in H_{0}^{1}(\Gamma), u_{2}, u_{3} \in H^{1}(\Gamma) \text { and } \\
\operatorname{div} u & =\frac{1}{r} \partial r u_{1}+\frac{1}{r} \partial_{\varphi} u_{2}+\partial_{z} u_{3}=0 ; \text { we set } \mathcal{V}=\widetilde{\mathcal{V}} \cap H_{0}^{1}(\Gamma)^{3} .
\end{aligned}
$$

Now let $E, \widetilde{E}$ be the $\mathcal{L}^{2}$-closures of $\mathcal{V}$ and $\widetilde{\mathcal{V}}$ respectively. We then have

Proposition 3.1. $E=\widetilde{E}$.

Remark. A proof is given in [17], Lemma 4.1; for a comparable situation in the Bénard-case see [19], Lemma 5.6.

We denote by $P$ the orthogonal projection from $\mathcal{L}^{2}(\Gamma)^{3}$ onto $E$.

Next we consider some Stokes operators related to MHD. With $\mathcal{V}$ as in (3.3) we stipulate:

$$
\begin{aligned}
& \operatorname{dom}\left(A_{0}\right)=H^{2}(\Gamma)^{3} \cap \mathcal{V} ; \text { if } w=(f, g, h) \in \operatorname{dom}\left(A_{0}\right) \\
& \text { then } A_{0} w=\nu P \Delta w=\nu P(\Delta f, \Delta g, \Delta h) .
\end{aligned}
$$

Proposition 3.2. $A_{0}$ is selfadjoint on $E$ and $<0$.

Remarks. For a proof see [22], III.1, III.2 (especially Thms. 1.5.4 and 2.1.1) where a much more general situation than ours is treated.

Now let $B, I_{1}, I_{2}$ be operators such that

$$
\begin{aligned}
& B w=\left(-\partial_{\varphi} g, \partial_{\varphi} f, 0\right), \quad I_{1} w=(f, 0,0), \quad I_{2} w=(f, g, 0) \\
& \text { where } \operatorname{dom}(B)=H^{1}(\Gamma)^{3}, \quad w=(f, g, h) .
\end{aligned}
$$

Proposition 3.3. The operator

$$
A_{S}=A_{0}-\nu P \frac{1}{r^{2}} I_{2}+\nu P \frac{2}{r^{2}} B, \quad \operatorname{dom}\left(A_{S}\right)=\operatorname{dom}\left(A_{0}\right)
$$


is selfadjoint and $<0$.

Proof. One easily shows that $A_{S}-A_{0}$ is symmetric and bounded relative to $A_{0}$ with relative bound zero, ie. for any $\varepsilon>0$ there is $C_{\varepsilon}$ such that

$$
\left\|\left(A_{S}-A_{0}\right) w\right\| \leq \varepsilon\left\|A_{0} w\right\|+C_{\varepsilon}\|w\|, \quad w \in \operatorname{dom}\left(A_{0}\right) ;
$$

see eg. [19], pg. 70 for a comparable situation. From this, the selfadjointness follows $([9]$, chpt. $5, \S 4)$.

In order to prove $A_{S}<0$, let $w=(f, g, h) \in \operatorname{dom}\left(A_{0}\right)$. A routine computation, based on the underlying Dirichlet conditions yields:

$$
\begin{aligned}
& \left\langle A_{S} w, w\right\rangle \leq-\int\left(|\partial f|^{2}+|\partial g|^{2}+|\partial h|^{2}\right) r d \mu \\
& -\int \frac{1}{r}\left(\left|\partial_{\varphi} f\right|^{2}+\left|\partial_{\varphi} g\right|^{2}+|f|^{2}+|g|^{2}+2 f^{*} \partial_{\varphi} g-2 g^{*} \partial_{\varphi} f\right) d \mu
\end{aligned}
$$

where $\int=\int_{\Gamma}, d \mu=d r d \varphi d z$. The claim now follows from the Poincaré inequality

$$
\int|f|^{2} r d \mu \leq c \int|\partial f|^{2} r d \mu, \quad \text { likewise with } g, h
$$

some $c=c\left(r_{1}, r_{2}\right)$, and the fact that the integrand in the second integral in (3.7) is $\geq 0$.

Remarks. $A_{S}$ is referred to as the Stokes operator of the fluid part of MHD, represented by the first three equation. In order to define a magnetic counterpart $A_{S}^{\prime}$ we formally define operators

$$
\widehat{\Delta}=\Delta-\frac{1}{r^{2}}, \quad \partial^{*} f=\frac{1}{r} \partial(r f)
$$

and note the relation

$$
\widehat{\Delta}=\partial \partial^{*}+\frac{1}{r^{2}} \partial_{\varphi}^{2}+\partial_{z}^{2}, \quad \Delta=\partial^{*} \partial+\frac{1}{r^{2}} \partial_{\varphi}^{2}+\partial_{z}^{2} .
$$

By a subscript we indicate the domain on which $\Delta$ resp. $\widehat{\Delta}$ is supposed to act ie. we stipulate:

$$
\operatorname{dom}\left(\Delta_{d}\right)=H^{2}(\Gamma) \cap H_{0}^{1}(\Gamma), \quad \operatorname{dom}\left(\Delta_{r}\right)=\widehat{H}^{2}(\Gamma), \quad \operatorname{dom}\left(\Delta_{n}\right)=\widetilde{H}^{2}(\Gamma)
$$

and likewise in case of $\widehat{\Delta}$. We then have

Proposition 3.4. (a) $\Delta_{d}, \Delta_{n}$ are selfadjoint, moreover $\Delta_{d}<0$ and $\Delta_{n} \leq 0$, (b) $\widehat{\Delta}_{r}$ is selfadjoint and $\leq 0$.

Proof. (a) is standard. As to (b) let $f \in \widehat{H}^{2}(\Gamma)$; we then have

$$
\int_{\Gamma} \widehat{\Delta}_{r} f \cdot f^{*} r d \mu=-\int_{\Gamma}\left(\left|\partial^{*} f\right|^{2}+\left|\frac{1}{r} \partial_{\varphi} f\right|^{2}+\left|\partial_{z} f\right|^{2}\right) r d \mu,
$$

what implies the symmetry of $\widehat{\Delta}_{r}$. The selfadjointness now follows from the easily provable fact that the equation $(\widehat{\Delta}-1) f=g$ has a unique weak solution $f \in H^{1}(\Gamma)$ for any $g \in \mathcal{L}^{2}(\Gamma)$, and from regularity arguments as put forward in [8], chpt. 8, [5] which show that $f \in \widehat{H}^{2}(\Gamma)$ 
Remark. $\Delta_{r}$, as a bounded perturbation of the selfadjoint $\widehat{\Delta}_{r}$, is of course also selfadjoint. Based on (3.2) we define the operators

$$
A_{1}=\eta \operatorname{diag}\left(\Delta_{d}, \widehat{\Delta}_{r}, \Delta_{n}\right), \quad A_{1}^{\prime}=\eta \operatorname{diag}\left(\widehat{\Delta}_{d}, \widehat{\Delta}_{r}, \Delta_{n}\right)
$$

which are selfadjoint by Prop. 3.4. Recalling (3.5), we now define an operator $A^{\prime}$ in terms of $A_{1}$ resp. $A_{1}^{\prime}$ according to

$$
A^{\prime}=A_{1}-\frac{\eta}{r^{2}} I_{1}+\frac{2 \eta}{r^{2}} B=A_{1}^{\prime}+\frac{2 \eta}{r^{2}} B, \quad \operatorname{dom}\left(A^{\prime}\right)=\operatorname{dom}\left(A_{1}\right) .
$$

It is easily seen that $A^{\prime}-A_{1}$ is bounded relative to $A_{1}$ with relative bound zero; this implies that $A^{\prime}$ is selfadjoint. An important property of $A^{\prime}$ is expressed by

Proposition 3.5. $P A^{\prime} \subseteq A^{\prime} P$, ie. $A^{\prime}$ commutes with $P$.

Outline of Proof. The property seems to be known but no explicit reference was found. For reasons of space we have to content us with an outline. The claim splits into two parts, the first of which states:

$$
\text { if } w \in \operatorname{dom}\left(A^{\prime}\right) \cap E \text { then } A^{\prime} w \in E \text {. }
$$

Now $v \in E$ iff $v \perp \nabla p$ for $p \in H^{1}(\Gamma)$; see Lemma 5.1 in [17] and Lemma 5.10 in [19]. Since the linear hull of elements $a b \varphi$ with $a \in C_{0}^{\infty}(R), b \in C_{\text {per }}^{\infty}([0,2 \pi])$, $\varphi \in C^{\infty}\left(\left[r_{1}, r_{2}\right]\right),(\partial \varphi)\left(r_{j}\right)=0, j=1,2$ is dense in $H^{1}(\Gamma)$, it suffices to show that if $w \in \operatorname{dom}\left(A^{\prime}\right) \cap E$ then $A^{\prime} w \perp \nabla(a b \varphi)$ for all $a, b, \varphi$ of the above type. The verification of this statement leads to lengthy but straightforward computation which we omit.

The second part of the claim is

$$
\text { if } w=(f, g, h) \in \operatorname{dom}\left(A^{\prime}\right) \text { then } P w \in \operatorname{dom}\left(A^{\prime}\right) .
$$

We sketch the proof. For $\phi \in H^{2}(\Gamma)$, let

$$
\widehat{\phi}(z)=\left(r_{2}-r_{1}\right)^{-1}(2 \pi)^{-1} \int_{r_{1}}^{r_{2}} \int_{0}^{2 \pi} \phi(r, \varphi, z) r d r d \varphi
$$

and consider the decomposition $w=w_{1}+w_{2}$ with $w_{2}=(0,0, \widehat{h}), w_{1}=w-w_{2}$. It is then easy to show that $P w_{2}=0$ and $w_{2} \in \operatorname{dom}\left(A^{\prime}\right)$. It thus remains to verify (3.15) with $w_{1}$ in place of $w$. We solve to this end the Neumann problem

$$
\Delta_{n} p=\operatorname{div} w_{1}=\partial^{*} f+\frac{1}{r} \partial_{\varphi} g+\partial_{z}(h-\widehat{h})=\phi .
$$

Since $\phi \in H^{1}(\Omega), \widehat{\phi}=0$, (3.11) has a unique solution $p \in H^{3}(\Omega)$ such that $\partial p \in H_{0}^{1}(\Omega), \widehat{p}=0$; this entails the relations

$$
\partial p \in H_{0}^{1}(\Omega), \partial r \cdot \frac{1}{r} \partial_{\varphi} p=\partial_{\varphi} \partial p \in H_{0}^{1}(\Omega), \partial \partial_{z} p=\partial_{z} \partial p \in H_{0}^{1}(\Omega) .
$$

By standard arguments on the other hand we have that

$$
P w_{1}=w_{1}-\nabla p=w_{1}-\left(\partial p, \frac{1}{r} \partial_{\varphi} p, \partial_{z} p\right)
$$


Since $p \in H^{3}(\Omega)$ and $w_{1} \in \operatorname{dom}\left(A^{\prime}\right)$ we infer from (3.17), (3.18) that $P w_{1} \in$ $\operatorname{dom}\left(A^{\prime}\right)$, proving (3.15). To conclude with, let $w, w_{1} \in \operatorname{dom}\left(A^{\prime}\right)$; by $(3.14),(3.15)$ we have the following equalities:

$$
\begin{aligned}
\left\langle P w_{1}, A^{\prime}(w-P w)\right\rangle & =\left\langle A^{\prime} P w_{1}, w-P w\right\rangle=\left\langle P A^{\prime} P w_{1}, w-P w\right\rangle= \\
& =\left\langle A^{\prime} P w_{1}, P(w-P w)\right\rangle=0 .
\end{aligned}
$$

Since the set $P w_{1}, w_{1} \in \operatorname{dom}\left(A^{\prime}\right)$ is dense in $E, P A^{\prime}(w-P w)=0$ follows. This implies

$$
P A^{\prime} w=P A^{\prime}(P w+w-P w)=P A^{\prime} P w=A^{\prime} P w,
$$

proving the claim.

Remarks. Besides Prop. 3.5 we have further invariance properties. For $v=$ $\left(v_{r}, v_{\varphi}, v_{z}\right), h=\left(h_{r}, h_{\varphi}, h_{z}\right)$, let

$$
\begin{aligned}
a^{\prime} & =\phi \partial_{z} v_{r}-\Omega \partial_{\varphi} h_{r}, \quad c^{\prime}=\phi \partial_{z} v_{z}-\Omega \partial_{\varphi} h_{z}-v_{r} \partial \phi \\
b^{\prime} & =h_{r} \partial V+\phi \partial_{z} v_{\varphi}-\Omega \partial_{\varphi} h_{\varphi}-\Omega h_{r}, \xi^{\prime}=\left(a^{\prime}, b^{\prime}, c^{\prime}\right) \\
\mathcal{N}_{1}^{\prime} & =(h \nabla) v_{r}-(v \nabla) h_{r}, \quad \mathcal{N}_{3}^{\prime}=(h \nabla) v_{z}-(v \nabla) h_{z}, \\
\mathcal{N}_{2}^{\prime} & =(h \nabla) v_{\varphi}-(v \nabla) h_{\varphi}-\frac{1}{r}\left(v_{\varphi} h_{r}-h_{\varphi} v_{r}\right), \quad \mathcal{N}^{\prime}=\left(\mathcal{N}_{1}^{\prime}, \mathcal{N}_{2}^{\prime}, \mathcal{N}_{3}^{\prime}\right)
\end{aligned}
$$

It can be shown that if $v, h \in H^{2}(\Gamma)^{3} \cap \widetilde{\mathcal{V}}$ then $\xi^{\prime}, \mathcal{N}^{\prime} \in \widetilde{\mathcal{V}} \subseteq E$. These invariance properties remove the difficulty that MHD has eight equations but only seven unknowns. Based on Prop. 3.5 we define the magnetic Stokes operator $A_{S}^{\prime}$ :

$$
\operatorname{dom}\left(A_{S}^{\prime}\right)=\operatorname{dom}\left(A^{\prime}\right) \cap \widetilde{\mathcal{V}}, \quad A_{S}^{\prime}=A^{\prime} \text { on } \operatorname{dom}\left(A_{S}^{\prime}\right)
$$

that is $A_{S}^{\prime}$ is the restriction of the selfadjoint operator $A^{\prime}$ to its invariant subspace $E$ and is thus itself selfadjoint on $E$.

Proposition 3.6. $A_{S}^{\prime} \leq 0$.

Remark. We postpone the proof of this statement which will be a corollary to our considerations on Bloch space. We now define the Stokes operator for the system MHD:

$$
\mathcal{A}=\operatorname{diag}\left(-A_{S},-A_{S}^{\prime}\right)=-\operatorname{diag}\left(A_{S}, A_{S}^{\prime}\right) .
$$

From Prop. 3.3, 3.6 and the remarks subsequent to (3.20) we infer

Proposition 3.7. $\mathcal{A}$ is selfadjoint on $E \times E$ and $\geq 0$.

In order to obtain a description of MHD as an abstract evolution equation we set

$$
\begin{aligned}
a^{\prime \prime} & =\phi \partial_{z} h_{r}-\Omega \partial_{\varphi} v_{r}+2 \Omega v_{\varphi}, \quad c^{\prime \prime}=h_{r} \partial \phi-\Omega \partial_{\varphi} v_{z}+\phi \partial_{z} h_{z} \\
b^{\prime \prime} & =\phi \partial_{z} h_{\varphi}-\Omega \partial_{\varphi} v_{\varphi}-\left(\frac{V}{r}+\partial V\right) v_{r}, \quad \xi=\left(a^{\prime \prime}, b^{\prime \prime}, c^{\prime \prime}\right) \\
\mathcal{N}_{1} & =(h \nabla) h_{r}-(v \nabla) v_{r}+\frac{v_{\varphi}^{2}}{r}=\frac{h_{\varphi}^{2}}{r}, \quad \mathcal{N}_{3}=(h \nabla) h_{z}-(v \nabla) v_{z}, \\
\mathcal{N}_{2} & =(h \nabla) h_{\varphi}-(v \nabla) v_{\varphi}+\frac{h_{\varphi} h_{r}}{r}-\frac{v_{\varphi} v_{r}}{r}, \quad \mathcal{N}=\left(\mathcal{N}_{1}, \mathcal{N}_{2}, \mathcal{N}_{3}\right) .
\end{aligned}
$$


In terms of (3.19), (3.22) we may cast the system MHD into the form

$$
\begin{aligned}
& \text { (a) } \partial_{t} v=A_{S} v+P \xi+P \mathcal{N}, \quad v \in \operatorname{dom}\left(A_{S}\right), h \in \operatorname{dom}\left(A_{S}^{\prime}\right), \\
& \text { (b) } \partial_{t} h=A_{S}^{\prime}+\xi^{\prime}+\mathcal{N}^{\prime}
\end{aligned}
$$

Due to Prop. 3.5 and the remarks following (3.14), no application of $P$ is needed in $(3.23)$, (b). Next we observe that $\xi=\xi(v, h), \xi^{\prime}=\xi^{\prime}(v, h)$ depend linearly on $v, h$ and thus induce a linear operator $L: H^{1}(\Gamma)^{6} \rightarrow \mathcal{L}^{2}(\Gamma)^{6}$ via

$$
L w=\left(\xi(v, h), \xi^{\prime}(v, h)\right) ; \quad w=(v, h)=\left(v_{r}, v_{\varphi}, v_{z}, h_{r}, h_{\varphi}, h_{z}\right) .
$$

We also write the nonlinearities $\mathcal{N}=\mathcal{N}(v, h), \mathcal{N}^{\prime}=\mathcal{N}^{\prime}(v, h)$ into a single one according to

$$
\mathcal{F}(w)=\left(\mathcal{N}(v, h), \mathcal{N}^{\prime}(v, h)\right), \quad w=(v, h)
$$

and extend the projection $\mathcal{P}$ to $\mathcal{L}^{2}(\Gamma)^{6}$ via

$$
\mathcal{P}=\operatorname{diag}(P, I), \quad I=\text { identity on } \mathcal{L}^{2}(\Gamma)^{3} .
$$

On the basis of $(3.16),(3.23)-(3.26)$ we can rewrite (3.23) as follows:

$$
\partial_{t} w+\mathcal{A} w=\mathcal{P} L w+\mathcal{P} \mathcal{F}(w), \quad w \in \operatorname{dom}(\mathcal{A}) .
$$

We interpret (3.27) as an evolution equation in fractional power spaces along established lines. Set $\mathcal{B}=\mathcal{A}+1, \mathcal{E}=E \times E$; by Prop. 3.7 we may define fractional power spaces $\mathcal{E}_{\alpha}$ via

$$
\mathcal{E}_{\alpha}=\operatorname{dom}\left(\mathcal{B}^{\alpha}\right), \quad\|\|_{\alpha}=\left\|\mathcal{B}^{\alpha}\right\| .
$$

For $\gamma \in\left(\frac{3}{4}, 1\right), w_{0} \in \mathcal{E}_{\gamma}$ there is a unique maximal solution $w \in C^{0}\left([0, T), \mathcal{E}_{\gamma}\right)$, $w(0)=w_{0}, T \leq \infty$ of $(3.27)$ with: (a) $w(t) \in \operatorname{dom}(\mathcal{A})$ for $t \in(0, T)$ and $\mathcal{A} w \in$ $C^{0}((0, T), \mathcal{E}),(\mathrm{b}) w \in C^{1}((0, T), \mathcal{E}),(\mathrm{c}) w(t)$ satisfies $(3.27)$ pointwise on $(0, T)$. If $w_{0} \in \operatorname{dom}(\mathcal{A})$, then $(0, T)$ in $(\mathrm{a})-(\mathrm{c})$ can be replaced by $[0, T)$. We refer to [13], pg. 196 for the general theory and to [20] for the comparable situation of the magnetic Bénard problem. The underlying inequalities valid for $\mathcal{F}(w)$ will be mentioned when needed.

\section{Ljapounov stability}

We now come to a discussion of the Ljapounov stability of the equlibrium solution in (1.2) which is based on three theorems, namely:

Theorem 1. If $a, b, \alpha, \beta$ in (1.2), (2.1) are sufficiently small then (3.27) is weakly energy stable, ie. if $w \in C^{0}\left([0, T), \mathcal{E}_{\gamma}\right), w(0) \in \operatorname{dom}(\mathcal{A})$ is a maximal solution of (3.27) then $\partial_{t}\|w(t)\|^{2} \leq 0, t \in[0, T)$.

Theorem 2. There are $k, C, \beta_{1}, \ldots, \beta_{n}, \varepsilon_{0}$ all $>0$ as follows. Let $|a|,|b|,|\alpha|,|\beta| \leq$ $\varepsilon_{0}$; if $w \in C^{0}\left([0, T), \mathcal{E}_{\gamma}\right), w(0) \in \operatorname{dom}(\mathcal{A})$ is a maximal solution of (3.27) then

$$
\begin{aligned}
\partial_{t}\left\|\mathcal{A}^{\frac{1}{2}} w(t)\right\|^{2}+k\left\|\mathcal{A}^{\frac{1}{2}} w(t)\right\|^{2} & \leq C\left\|\mathcal{A}^{\frac{1}{2}} w(t)\right\|^{6}+C\left\|\mathcal{A}^{\frac{1}{2}} w(t)\right\|^{4} \\
& +C\left(\|w(t)\|^{\beta_{1}}+\cdots+\|w(t)\|^{\beta_{n}}\right)
\end{aligned}
$$


for $t \in[0, T)$.

The proof of Thm. 1 is postponed. Prior to prove Thm. 1 we show how to infer Ljapounov stability from Thm. 1, 2 .

Theorem 3. Let $|a|,|b|,|\alpha|,|\beta| \leq \varepsilon_{0}$. Given $\varepsilon>0$ there is $\delta_{0}>0$ as follows: if $w_{0} \in \operatorname{dom}(\mathcal{A})$ and $\left\|w_{0}\right\|_{H^{1}} \leq \delta_{0}$ then the associated maximal solution $w \in$ $C^{0}\left([0, T), \mathcal{E}_{\gamma}\right), w(0)=0$ is global and satisfies $\|w(t)\|_{H^{1}} \leq \varepsilon, t \geq 0$.

Remark. The difficult part is provided by the proof of Thm. 1 which will be given in detail. The proof of Thm. 2, while lengthy when worked out in detail, is just an amplification of the proof of Lemma 2 in [16] and is considered as routine by experts. In order to keep the length of the paper within limits we therefore state Thm. 2 without proof and refer to [14], [16] for corresponding proofs in the context of the MBP and Navier Stokes. It seems however justified to go briefly into the mechanism which ties Thms. 1, 2 with Thm. 3. This step is based on two auxiliary propositions of routine character which we state without proof for reason of space:

Proposition 4.1. Let $k, C>0$ be given. For $\varepsilon>0$ we find $\delta>0$ as follows: if $h \in C^{1}\left([0, T), R_{+}\right), \psi \in C^{0}\left([0, T), R_{+}\right)$satisfy

$$
\partial_{t} h+k h \leq C h^{3}+C h^{2}+C \psi, \quad t \in[0, T),
$$

and if $h(0) \leq \delta$ and $\psi(t) \leq \delta, t \in[0, T)$ then $h(t) \leq \varepsilon, t \in[0, T)$.

Remark: For a proof we refer to the proof of Prop. 6.1 in the Appendix of [16] which applies to Prop. 4.1 without change.

Proposition 4.2. There are $c_{1}, c_{2}$ as follows: if $w \in \operatorname{dom}(\mathcal{A})$ then

$$
\|w\|_{H^{1}} \leq c_{1}\left(\|w\|+\left\|\mathcal{A}^{\frac{1}{2}} w\right\|\right) \leq c_{2}\|w\|_{H^{1}} .
$$

Proof of Thm. 3. With $k, C$ as in Thm. 2 we associate inequality (4.2). Fix $\varepsilon \in(0,1)$ and let $\delta \leq \varepsilon$ be related to $\varepsilon$ via Prop. 4.1. By Prop. 4.2 there is $\delta_{0} \leq \delta$ such that $w(0) \in \operatorname{dom}(\mathcal{A}),\|w(0)\|_{H^{1}} \leq \delta_{0}$ implies

$$
\begin{aligned}
& \left\|\mathcal{A}^{\frac{1}{2}} w(0)^{2}\right\| \leq \delta, \quad\|w(0)\|^{\beta_{1}}+\cdots+\|w(0)\|^{\beta_{n}} \leq \delta, \\
& \beta_{j} \text { as in Thm. 2. }
\end{aligned}
$$

Now fix $w_{0} \in \operatorname{dom}(\mathcal{A})$ with $\left\|w_{0}\right\|_{H^{1}} \leq \delta_{0}$ and let $w \in C^{1}\left([0, T), \mathcal{E}_{\gamma}\right), w(0)=w_{0}$ be the associated maximal solution; set

$$
h(t)=\left\|\mathcal{A}^{\frac{1}{2}} w(t)\right\|^{2}, \quad \psi(t)=\|w(t)\|^{\beta_{1}}+\cdots+\|w(t)\|^{\beta_{n}} .
$$

By Thm. $2, h(t), \psi(t)$ satisfy inequality $(4.2)$ on $[0, T)$. Now $h(0), \psi(0) \leq \delta$ by construction whence $\psi(t) \leq \delta$ for $t \in[0, T)$ by Thm. 1 . By Prop. 4.1 and Thm. 1 we thus have

$$
\left\|\mathcal{A}^{\frac{1}{2}} w(t)\right\|^{2} \leq \varepsilon, \quad\|w(t)\| \leq \varepsilon, \quad t \in[0, T) .
$$


From Prop. 4.2 and (4.4) we infer

$$
\|w(t)\|_{H^{1}} \leq 2 c_{1} \varepsilon^{\frac{1}{2}}, \quad t \in[0, T) .
$$

By classical results on evolution equations of Navier-Stokes type, (4.5) implies that the solution $w()$ is global $(T=\infty)$ and that (4.5) holds for $t \geq 0$; for proofs of this fact which apply to the present situation see the proof of Thm. 4 in [16]. The statement now follows after a relabeling of $\varepsilon$

Corollary. Given $\varepsilon$ there is $\delta$ as follows: if $w_{0} \in \operatorname{dom}(\mathcal{A}),\left\|w_{0}\right\|_{H^{1}} \leq \delta$, and if $w \in C^{0}\left([0, T), \mathcal{E}_{\gamma}\right), w(0)=w_{0}$ is the associated maximal solution then it is global and $\|w(t)\|_{\gamma} \leq \varepsilon, t \geq 1$.

Remark. The corollary follows from Thm. 3 by means of the singular Gronwall inequality in [1], pg. 52-53 by using the arguments in the proofs of Thms. 2, 3 in [16] as they stand.

\section{Orr-Sommerfeld equations}

As is apparent from sect. 4 , Thm. 3 is an immediate consequence of Thms. 1, 2 . Now while the proof of Thm. 2 is a lengthy but rather straightforward elaboration of the proof of lemma 2 in [16] (and thus omitted) the proof of Thm. 1 is more difficult. In fact it is concerned with the perturbation theory of the continuous spectrum of a selfadjoint operator, a topic which is far less complete than the perturbation theory of discrete spectra. There are however situations in hydrodynamics in which one can describe the continuous spectrum by a parametrised family of discrete spectra, thus reducing the perturbation theory to that of discrete spectra. These are described by the so called Orr-Sommerfeld equations. The bridge which justifies this passage from the continuous to the discrete spectrum is provided by Bloch space theory, described in details in two books [19], [25] and a series of papers [12], [15], [17], [18], [20], [21]. For lack of space we cannot go into Bloch space theory, but refer to the cited literature instead. Here we restrict us to describe the Orr-Sommerfeld equations to which the proof of Thm. 1 gives rise.

First, recall $A_{S}^{\prime}$ given by (3.2), (3.12), (3.13), (3.20). Let $M(r)=\left(m_{j k}(r)\right)$, $j, k \leq 3, r \in\left[r_{1}, r_{2}\right]$ be a real symmetric $3 \times 3$ matrix with smooth entries. $M(r)$ induces a bounded operator $M$ on $\mathcal{L}^{2}(\Omega)^{3}$ in the obvious way:

$$
(M h)(r)=M(r) h(r), \quad h \in \mathcal{L}^{2}(\Gamma)^{3} .
$$

Based on the remarks prior to Prop. 3.6 and on Prop. 3.6 we have by standard functional anaysis:

Proposition 5.1. $A_{S}^{\prime}+\mathcal{P} M$ is selfadjoint on $E$ and bounded from above.

The matrices $M(r)$ in the proof of Thm. 1 are expressed in a simple way in terms of the equilibria in (1.2) and depend smoothly on $a, b, \alpha, \beta$, ie. $M(r)=$ 
$M(a, b, \alpha, \beta, r)$ whereby $M(0,0,0,0, r)=0$. The main task then is to show:

if $a, b, \alpha, \beta$ are small then $\sigma \leq 0$ for $\sigma \in \sigma\left(A_{S}^{\prime}+\mathcal{P} M(a, b, \alpha, \beta, \cdot)\right)$.

By Prop. 3.6, (5.2) holds if $a=b=\alpha=\beta=0$. In order to handle (5.2), we invoke a technical step which amounts to replace the continuous spectrum $\sigma\left(A_{S}^{\prime}+\mathcal{P} M\right)$ by an equivalent set of discrete spectra, associated with the so called Orr-Sommerfeld equations. In order to describe them we pick a period $L>0$, arbitrarily but fixed, let $\theta \in[0,2 \pi], \alpha, k \in \mathbb{Z}$ and define

$$
\widehat{\alpha}=\widehat{\alpha}(\theta)=(2 \pi \alpha+\theta) L^{-1} .
$$

With $\partial=\partial_{r}$ we set

$$
\mathcal{D}=\partial^{*} \partial, \quad \widehat{\mathcal{D}}=\mathcal{D}-\frac{1}{r^{2}}=\partial \partial^{*}, \quad \partial^{*} f=r^{-1} \partial(r f) .
$$

We also need function spaces:

$$
\begin{aligned}
& f \in \widehat{H}^{2}(I) \text { iff } f \in H^{2}(I) \text { and } \partial(r f) \in H_{0}^{1}(I), \\
& f \in \widetilde{H}^{2}(I) \text { iff } f \in H^{2}(I) \text { and } \partial f \in H_{0}^{1}(I),
\end{aligned}
$$

where $I=\left[r_{1}, r_{2}\right]$. Recalling $M(r)$ prior to (5.1) we now consider the following eigenvalue problem. If $\theta \in[0,2 \pi]$ and $\widehat{\alpha}^{2}+k^{2}>0$, we seek $h_{1} \in H^{2}(I) \cap H_{0}^{1}(I)$, $h_{2} \in \widehat{H}^{2}(I), h_{3} \in \widetilde{H}^{2}(I)$ (not all $\left.=0\right), p \in H^{1}(I)$ and $\sigma \in \mathbb{C}$ such that

(i) $\quad \eta\left(\mathcal{D} h_{1}-\left(\frac{k^{2}}{r^{2}}+\widehat{\alpha}^{2}\right) h_{1}-\frac{h_{1}}{r^{2}}-\frac{2 i k}{r^{2}} h_{2}\right)+\Sigma m_{1 j} h_{j}+\partial p=\sigma h_{1}$

(ii) $\quad \eta\left(\widehat{\mathcal{D}} h_{2}-\left(\frac{k^{2}}{r^{2}}+\widehat{\alpha}^{2}\right) h_{2}+\frac{2 i k}{r^{2}} h_{1}\right)+\Sigma m_{2 j} h_{j}+\frac{i k p}{r}=\sigma h_{2}$

(iii) $\quad \eta\left(\mathcal{D} h_{3}-\left(\frac{k^{2}}{r^{2}}+\widehat{\alpha}^{2}\right) h_{3}\right)+\Sigma m_{3 j} h_{j}+i \widehat{\alpha} p=\sigma h_{3}$

(iv) $\quad \partial^{*} h_{1}+\frac{i k h_{2}}{r}+i \widehat{\alpha} h_{3}=0$

If $\theta \in\{0,2 \pi\}$ and $\widehat{\alpha}^{2}+k^{2}=0$ then we seek $h_{1}, h_{2}, h_{3}, p$ as above, $\sigma \in \mathbb{C}$ and a constant $c \in \mathbb{C}$ such that (i), (ii), (iv) hold but with (iii) now replaced by

$$
\eta \mathcal{D} h_{3}+\Sigma m_{3 j} h_{j}+c=\sigma h_{3}, \quad \int_{r_{1}}^{r_{2}} h_{3} r d r=0 .
$$

Remarks. Eq. (5.6), (5.7) are obtained from the last four MHD-equations in sect. 2 by straightforward Fourier expansion. Orr-Sommerfeld equations are widely used; see eg. [2], [3], [7], [11] in case of CT, [2], [7], [11], [24] in case of BP, [2], [15] in case of MBP and [2] in case of MCT. As to the structure of (5.6), (5.7) we have the following proposition which is proved by standard arguments:

Proposition 5.2. Given $\alpha, k \in \mathbb{Z}, \theta \in[0,2 \pi]$, the associated eigenvalue problem (5.6), (5.7) is (after elimination of p) selfadjoint, the set of eigenvalues $\sigma$ real, discrete and bounded from above

In order to describe the connection between the family of eigenvalue problems (5.6), (5.7) and the original problem (5.2) we need 
Definition 1. Let $\theta \in[0,2 \pi]$. Then $\sigma \in \Sigma(\theta)$ iff there are $\alpha, k \in \mathbb{Z}$ such that the eigenvalue problem (5.6), (5.7) (with $\alpha, k, \sigma$ therein) admits a nontrivial solution $h_{1}, h_{2}, h_{3}, p$

Recalling the operator $A_{S}^{\prime}+\mathcal{P} M$ in Prop. 5.1 we have

Theorem 4.

$$
\begin{aligned}
\sigma\left(A_{S}^{\prime}+\mathcal{P} M\right) & =\bigcup_{\theta \in[0,2 \pi]} \Sigma(\theta) \\
& =\operatorname{closure}\left(\bigcup_{\theta \in(0,2 \pi)} \Sigma(\theta)\right)
\end{aligned}
$$

Remarks. We briefly comment on the proof of Thm. 4. In practice a proof like that of Thm. 4 is accomplished in a single step, either via partial Fourier transform or via Fourier series. See [26] for an application of the partial Fourier transform to plane parallel Couette flow and to [2] for a more formal application of Fourier series to the magnetic Couette-Taylor flow. Our Thm. 4 is essentially a corollary of Bloch space theory, well known in the context of Schrödinger equations with periodic potentials $([25])$. For Stokes operators much more general than those considered here, Bloch space theory is worked out in [15], [17]-[20] for the Boussinesq and the magnetic Boussinesq equations. The domain underlying the analysis in [15], [17]-[20] is the infinite layer, but it extends without change to any reasonable domain having at least one translation invariant direction as emphasized in the introductions of [17], [18], [19].

The spectral formulas in Thms. 2.2, 2.3 of [20] remain valid when rephrased in terms of cylindrical coordinates. The formula in Thm. 2.3, [20] even simplifies, due to the fact that the domain in (1.1) is only translation invariant in the $z$-direction,; see [17], [18] in this respect. Theorem 4 then follows from these spectral formulas by standard Fourier analysis. (see [3], [17], [24] for details). Bloch space theory for the domain in (1.1) is worked out in [17] (see also [18]) and for more general cylindrical domains in [12]. The advantage of a proof via Bloch space theory is that it extends to the case where the equilibrium solution is $L$-periodic and not constant in $z$, a case where the method of partial Fourier transform fails.

\section{Weak energy stability}

It remains to prove Thm. 1 . It is here where we have to make use of Thm. $4,4^{\prime}$, 5 in section 5 . The proof will be given in steps. In order to keep the presentation in limits, we will omit lengthy routine computations or present them in condensed form. We proceed in steps $\mathrm{S} 1, \mathrm{~S} 2, \ldots$ We first define a linear operator $L_{2}$ on $H_{1}(\Gamma)^{6}$ which acts on $w=(v, h)$ with $v=\left(v_{r}, v_{\varphi}, v_{z}\right), h=\left(h_{r}, h_{\varphi}, h_{z}\right)$ as follows:

$$
L_{2} w=\left(2 \Omega v_{\varphi},-(\Omega+\partial V) v_{r}, h_{r} \partial \phi,(\partial V-\Omega) h_{r},-v_{r} \partial \phi\right) .
$$


Here $\Omega, V, \phi$ are as in (1.2), (2.1) resp.. Now let a solution $w \in C^{0}\left([0, T), E_{\gamma}\right)$, $w(0) \in \operatorname{dom}(\mathcal{A})$ of $(3.27)$ be given.

Step S1: We multiply (3.27) scalarly with $w=w(t)$. By a lengthy but straightforward computation we end up with the equation

$$
\frac{1}{2} \partial_{t}\|w\|^{2}=\langle-\mathcal{A} w, w\rangle+\left\langle L_{2} w, w\right\rangle=J(w),
$$

with $L_{2}$ as in (6.0). According to (1.2), (2.1) we have

$$
\Omega-\partial V=\frac{2 b}{r^{2}}, \quad \partial \phi=\frac{\beta}{r} .
$$

We also recall $\mathcal{A}$ as given by (3.21). Since $w=(v, h)$, with $v=\left(v_{r}, v_{\varphi}, v_{z}\right)$ in $\operatorname{dom}\left(A_{S}\right)$ and $h=\left(h_{r}, h_{\varphi}, h_{z}\right)$ in $\operatorname{dom}\left(A_{S}^{\prime}\right)$ we can rewrite $J(w)$ in (6.1) as follows:

$$
\begin{aligned}
J(w) & =\left\langle A_{S} v, v\right\rangle+\left\langle A_{S}^{\prime} h, h\right\rangle+2 b \int \frac{v_{r} v_{\varphi}}{r^{2}} d_{\mu} \\
& -2 b \int \frac{h_{r} h_{\varphi}}{r^{2}} d \mu+\beta \int \frac{h_{r} v_{z}-h_{z} v_{r}}{r} d \mu,
\end{aligned}
$$

where $\int=\int_{\Gamma}, d \mu=r d r d \varphi d z$. In this first step we assume: (I) $\boldsymbol{\beta}=\mathbf{0}$. We set

$$
\begin{aligned}
& J_{0}(v)=\left\langle A_{S} v, v\right\rangle+2 b \int \frac{v_{r} v_{\varphi}}{r^{2}} d \mu, \quad v \in \operatorname{dom}\left(A_{S}\right), \\
& J_{1}(h)=\left\langle A_{S}^{\prime} h, h\right\rangle-2 b \int \frac{h_{r} h_{\varphi}}{r^{2}} d \mu, \quad h \in \operatorname{dom}\left(A_{S}^{\prime}\right) .
\end{aligned}
$$

Since $\left\langle A_{S} v, v\right\rangle \leq-c_{0}\|v\|^{2}$ for some $c_{0}=c_{0}(\nu)$ by Prop. 3.3 we immediately get

Proposition 6.1. There is $c_{1}=c_{1}(\nu)>0$ and $\delta=\delta(\nu)>0$ such that $|b| \leq \delta$ entails: $J_{0}(v) \leq-c_{1}\|v\|^{2}, v \in \operatorname{dom}\left(A_{S}\right)$.

By virtue of (6.1)-(6.3), Theorem 1 follows immediately from the crucial

Lemma 6.1. There is $\delta=\delta(\eta)>0$ such that $|b| \leq \delta$ implies $J_{1}(h) \leq 0, h \in$ $\operatorname{dom}\left(A_{S}^{\prime}\right)$

The proof is split into propositions. We define a $3 \times 3$-matrix $M$ according to

$$
\begin{aligned}
& M=M(b, r)=\left(m_{j k}\right), \quad m_{12}=m_{21}=-\frac{b}{r^{2}} ; \\
& m_{j k}=0 \text { otherwise. }
\end{aligned}
$$

The bounded operator induced by $M$ via (5.1) is also denoted by $M$. Since $A_{S}^{\prime}+$ $\mathcal{P} M$ is selfadjoint, Lemma 6.1 is proved if we can show for small $|b|$ :

$$
\text { if } \sigma \in \sigma\left(A_{S}^{\prime}+\mathcal{P} M\right) \text { then } \sigma \leq 0 \text {. }
$$

We now combine (6.5) with Thm. 4 so as to obtain

Lemma 6.1'. $J_{1}(h) \leq 0, h \in \operatorname{dom}\left(A_{S}^{\prime}\right)$ iff for all $\theta \in(0,2 \pi), k, \alpha \in \mathbb{Z}$ the associated eigenvalue problem (5.6) admits only eigenvalues $\sigma \leq 0$, whereby $M=$ $\left(m_{j k}\right)$ is given by (6.4). 
Lemma $6.1^{\prime}$ now leads to a distinction of cases, namely the cases $k=0,|k|=1$ or $|k| \geq 2$. The easiest case is $|k| \geq 2$.

Proposition 6.2. If $|k| \geq 2,|b| \leq 2 \eta$ then the eigenvalue problem (5.6) has only eigenvalues $\sigma \leq 0$ for any choice of $\alpha \in \mathbb{Z}, \theta \in(0,2 \pi)$.

Proof. With $M$ in (5.6) given by (6.4), we fix $\theta \in(0,2 \pi), \alpha, k \in \mathbb{Z}$ with $|k| \geq 2$. We let $h_{1}=h_{r}, h_{2}=h_{\varphi}, h_{3}=h_{z}, p$ be a solution of (5.6) and set $h=\left(h_{r}, h_{\varphi}, h_{z}\right)$; we may assume

$$
\|h\|^{2}=\int\left(\left|h_{r}\right|^{2}+\left|h_{\varphi}\right|^{2}+\left|h_{z}\right|^{2}\right) r d r=1 .
$$

We also define quadratic forms

$$
\begin{aligned}
& Q_{0}(h)=\left(k^{2}+1\right)\left|h_{r}\right|^{2}+k^{2}\left|h_{\varphi}\right|^{2}+2 i k h_{\varphi} h_{r}^{*}-2 i k h_{r} h_{\varphi}^{*}, \\
& Q_{1}(h)=h_{\varphi} h_{r}^{*}+h_{r} h_{\varphi}^{*} .
\end{aligned}
$$

We then multiply (5.6) scalarly with $h$, take the divergence condition in (5.6) into account and recall the boundary conditions associated with the spaces $H^{2}(I) \cap$ $H_{0}^{1}(I), \widehat{H}_{0}^{1}(I), \widetilde{H}^{2}(I)$. By a straightforward computation we find

$$
\begin{aligned}
\sigma= & -\eta \int\left(\left|\partial h_{r}\right|^{2}+\left|\partial^{*} h_{\varphi}\right|^{2}+\left|\partial h_{z}\right|^{2}\right) r d r-\eta k^{2} \int \frac{\left|h_{z}\right|^{2}}{r^{2}} r d r \\
& -\eta \widehat{\alpha}^{2}-\int \frac{1}{r^{2}}\left(\eta Q_{0}(h)+b Q_{1}(h)\right) r d r
\end{aligned}
$$

where $\int=\int_{I}, I=\left[r_{1}, r_{2}\right]$. The righthand side of this equation is $\leq 0$ if the quadratic form $\eta Q_{0}(h)+b Q_{1}(h)$ is $\geq 0$, and this holds if its determinant $d_{k}$ is $\geq 0$.

Now $d_{k}$ is given by

$$
d_{k}=\eta^{2}\left(k^{4}+k^{2}\right)-\left(4 \eta^{2} k^{2}+b^{2}\right) .
$$

Since $|k| \geq 2$ we have $d_{k} \geq 0$ for $|b| \leq 2 \eta$

Step 2: Next we come to the case $k=1$ which is more involved. Its discussion requires an auxiliary operator $A_{\lambda},(\lambda \in R)$. We provide $\mathcal{L}^{2}(I)$ with the scalar product $(f, g)=\int f g^{*} r d r$ and let $\langle$,$\rangle be the induced scalar product on \mathcal{L}^{2}(I) \times$ $\mathcal{L}^{2}(I)$. With $\widehat{H}^{2}(I), \widetilde{H}^{2}(I)$ as in (5.5) we stipulate:

$$
\begin{aligned}
\operatorname{dom}\left(A_{\lambda}\right) & =\left(H^{2}(I) \cap H_{0}^{1}(I)\right) \times \widehat{H}^{2}(I) ; \text { for } h=\left(h_{r}, h_{\varphi}\right) \in \operatorname{dom}\left(A_{\lambda}\right), \\
A_{\lambda} h & =\left(\widehat{D} h_{r}-\frac{\lambda^{2}}{r^{2}} h_{r}-\frac{2 i \lambda}{r^{2}} h_{\varphi}, \widehat{D} h_{\varphi}-\frac{\lambda^{2}}{r^{2}} h_{\varphi}+\frac{2 i \lambda}{r^{2}} h_{r}\right),
\end{aligned}
$$

with $D, \widehat{D}$ as in (5.4). It is easily seen that $A_{\lambda}$ is selfadjoint, is bounded from above and has compact resolvents. Minor computations are needed to prove

Proposition 6.3. (a) If $h=\left(h_{r}, h_{\varphi}\right) \in \operatorname{dom}\left(A_{\lambda}\right)$ and $\partial^{*} h_{r}+\frac{i \lambda}{r} h_{\varphi}=0$ then 
$\left\langle A_{\lambda} h, \nabla p\right\rangle=0$ for $p \in H^{1}(I)$, (b) if $p \in H^{3}(I) \cap \widetilde{H}^{2}(I)$ then

$$
\nabla\left(\mathcal{D} p-\frac{\lambda^{2}}{r^{2}} p\right)=A_{\lambda} \nabla p, \quad \text { where } \quad \nabla f=\left(\partial f, \frac{i \lambda}{r} f\right) .
$$

Hint of proof. One first assumes $p \in H^{3}(I) \cap \widetilde{H}^{2}(I)$ and shows by a straightforward computation that $\left\langle A_{\lambda} h, \nabla p\right\rangle=0$; since $H^{3}(I) \cap \widetilde{H}^{2}(I)$ is dense in $H^{1}(I)$, clause (a) follows by approximation. As to (b) one notes that by (5.5), (6.6) we have $\nabla p \in \operatorname{dom}\left(A_{\lambda}\right)$ if $p \in H^{3}(I) \cap \widetilde{H}^{2}(I)$; the claim of (b) then follows by direct computation

Proposition 6.4. Let $\lambda \neq 0, p \in H^{3}(I) \cap \widetilde{H}^{2}(I)$ and $A_{\lambda} \nabla p=0$; then $p=0$.

Proof. By (b) of Prop. 6.3 and our assumptions we have that

$$
\frac{i \lambda}{r}\left(\partial^{*} \partial p-\frac{\lambda^{2}}{r^{2}} p\right)=0 \quad \text { ie. } \quad \partial^{*} \partial p=\frac{\lambda^{2}}{r^{2}} p .
$$

Since $p \in \widetilde{H}^{2}(I)$ we infer from (6.7):

$$
\int \partial^{*} \partial p p^{*} r d r=-\int|\partial p|^{2} r d r=\lambda^{2} \int \frac{|p|^{2}}{r^{2}} r d r, \quad \int=\int_{I}
$$

whence $p=0$ as claimed.

Of similar type is

Proposition 6.5. Let $h=\left(h_{r}, h_{\varphi}\right) \in \operatorname{dom}\left(A_{\lambda}\right)$ such that $A_{\lambda} h=0$ and $\operatorname{div} h=$ $\partial^{*} h_{r}+\frac{i \lambda}{r} h_{\varphi}=0$. If $\lambda \neq 0$ then $h=0$.

Proof. Let $A_{\lambda} h=(f, g)$ with $f, g$ expressed in terms of $h_{r}, h_{\varphi}$ via (6.6). We recall $\widehat{D}=\partial \partial^{*}$ and combine the equation $f=0$ with the assumption $\operatorname{div} h=0$ so as to get:

$$
\frac{2 i \lambda}{r^{2}}=2 \partial\left(\frac{h p}{r}\right)+\frac{4 h_{\varphi}}{r^{2}} .
$$

We substitute the righthand side of (6.8) for $\frac{2 i \lambda}{r^{2}} h_{r}$ into the equation $g=0$ so as to obtain:

$$
\partial \frac{1}{r} \partial Y-\frac{\lambda^{2} Y}{r^{3}}+\frac{2 \partial Y}{r^{2}}=0, \quad Y=r h_{\varphi} .
$$

Now (6.9) can be rewritten as

$$
\partial r \partial Y=\frac{\lambda^{2} Y}{r} .
$$

Since $Y=r h_{\varphi}$ is in $\widetilde{H}^{2}(I)$ by (5.5), we get from (6.10) by partial integration:

$$
-\int|\partial Y|^{2} d r=\lambda^{2} \int \frac{|Y|^{2}}{r} d r
$$

whence $Y=0$ and hence $h_{\varphi}=h_{r}=0$ 
The major conclusion to be drawn from Prop. 6.4-6.6 is

Proposition 6.6. Let $h \in \operatorname{dom}\left(A_{\lambda}\right)$ and $\lambda \neq 0$. If $A_{\lambda} h=0$ then $h=0$.

Proof. We first solve the Neumann problem

$$
\mathcal{D} p-\frac{\lambda^{2}}{r^{2}} p=\operatorname{div} h, \quad p \in \widetilde{H}^{2}(I), \quad\left(\mathcal{D}=\partial^{*} \partial\right)
$$

and note that since $\operatorname{div} h \in H^{1}(I)$, (6.11) has a unique solution such that:

$$
\text { (a) } \quad p \in H^{3}(I) \cap \widetilde{H}^{2}(I), \quad\left(\text { b) } \nabla p \in \operatorname{dom}\left(A_{\lambda}\right),(\text { c) } \operatorname{div}(h-\nabla p)=0 .\right.
$$

From the assumption $A_{\lambda} h=0$ we infer

$$
A_{\lambda}(h-\nabla p)+A_{\lambda} \nabla p=0 .
$$

From (6.12)(a)-(c) and Prop. 6.3 it follows that $A_{\lambda} \nabla p$ is orthogonal to $A_{\lambda}(h-\nabla p)$ whence

$$
\text { (a) } A_{\lambda}(h-\nabla p)=0, \quad \text { (b) } \quad A_{\lambda} \nabla p=0
$$

by (6.13). From $(6.12)(\mathrm{c}),(6.14)(\mathrm{b})$ and Prop. 6.4 on the other hand we obtain $p=0$. This, together with $(6.12)(\mathrm{c}),(6.14)(\mathrm{a})$ and Prop. 6.5 entails $h=0$

Prop. 6.7 leads us to the last remark prior to the settlement of the case $k=1$.

Proposition 6.7. Given $\lambda \neq 0$ there is $c(\lambda)>0$ such that $A_{\lambda} \leq-c(\lambda)$.

Proof. Let $Q_{\lambda}(x, y)=\left(1+\lambda^{2}\right)|x|^{2}+\lambda^{2}|y|^{2}+2 i \lambda y x^{*}-2 i \lambda x y^{*}$. By elementary arguments we find $c_{0}>0$ such that

$$
Q_{\lambda}(x, y) \geq c_{0}\left(|x|^{2}+|y|^{2}\right), \quad|\lambda| \geq 2 .
$$

Next let $h=\left(h_{r}, h_{\varphi}\right) \in \operatorname{dom}\left(A_{\lambda}\right)$ and recall (6.6). Based on (6.15) and by proceeding as in the proof of Prop. 6.2 we conclude

$$
\begin{aligned}
\left\langle A_{\lambda} h, h\right\rangle & =-\int\left(\left|\partial h_{r}\right|^{2}+\left|\partial^{*} h_{\varphi}\right|^{2}\right) r d r-\int \frac{1}{r} Q_{\lambda}\left(h_{r}, h_{\varphi}\right) d r \\
& \leq-c_{0} \int \frac{1}{r}\left(\left|h_{r}\right|^{2}+\left|h_{\varphi}\right|^{2}\right) d r \leq-c_{1} \int\left(\left|h_{r}\right|^{2}+\left|h_{\varphi}\right|^{2}\right) r d r
\end{aligned}
$$

That is we have

$$
\text { if }|\lambda| \geq 2 \text { and } \sigma \in \sigma\left(A_{\lambda}\right) \text { then } \sigma \leq-c_{1}
$$

Now let the proposition be false. Then one finds $\lambda_{0} \neq 0$ and $\sigma_{0} \in \sigma\left(A_{\lambda_{0}}\right)$ for some $\sigma_{0} \geq 0$; by Prop. 6.5 necessarily have $\sigma_{0}>0$ Assume e.g. $\lambda_{0}>0$. Sets $S_{1}, S_{2} \subseteq(0, \infty)$ are then defined according to: (a) $\lambda \in S_{1}$ iff there is $\sigma \in \sigma\left(A_{\lambda}\right)$ with $\sigma>0$, (b) $\lambda \in S_{2}$ iff all eigenvalues of $\sigma\left(A_{\lambda}\right)$ are $<0$. From (6.17), Prop. 6.6 and since $\lambda_{0} \in S_{1}$ we thus infer:

$$
S_{1} \neq \emptyset, S_{2} \neq \emptyset, S_{1} \cap S_{2}=\emptyset, S_{1} \cup S_{2}=(0, \infty) .
$$


Since on the other hand each $A_{\lambda}$ has pure point spectrum it follows from classical results of perturbation theory ([10] Thm. 3.16, pg. 212, [13]) that $S_{1}, S_{2}$ are open. But this contradicts (6.18). Thus a $\lambda_{0}>0$ as assumed cannot exist. If $\lambda_{0}<0$ we apply a similar reasoning to $(-\infty, 0)$. From this arguments the proposition follows.

Prop. 6.7 allows us to settle the case $|k|=1$ in Lemma $1^{\prime}$ :

In fact we have:

Proposition 6.8. Let $|k|=1$. If $2|b| \leq r_{1}^{2} \eta \min (c(1), c(-1))$ then the eigenvalue problem (5.6) has only eigenvalues $\sigma \leq 0$ for any $\theta \in(0,2 \pi), \alpha \in \mathbb{Z}$.

Proof. Let $h=\left(h_{r}, h_{\varphi}, h_{z}\right), p$ be a solution $\neq 0$ of (5.6) for some $\theta \in(0,2 \pi)$, $\alpha \in \mathbb{Z}$ and $|k|=1$; we may assume $\|h\|=1$. We multiply the first three equations of (5.6) scalarly with $h$ and take care of the divergence condition in (5.6) and of $\|h\|=1$. By straightforward computation we get:

$$
\begin{aligned}
\sigma \leq & -\eta \int\left(\left|\partial^{*} h_{r}\right|^{2}+\left|\partial^{*} h_{\varphi}\right|^{2}+\left|\partial h_{z}\right|^{2}\right) r d r+2 i k \int\left(h_{r} h_{\varphi}^{*}-h_{\varphi} h_{r}^{*}\right) \frac{d r}{r} \\
& -\eta \int\left(\left|h_{r}\right|^{2}+\left|h_{\varphi}\right|^{2}+\left|h_{z}\right|^{2}\right) \frac{d r}{r}-b \int\left(h_{\varphi} h_{r}^{*}+h_{r} h_{\varphi}^{*}\right) \frac{d r}{r} .
\end{aligned}
$$

We now set $c_{1}=\min (c(1), c(-1))$ with $c(\lambda)$ as in Prop. 6.7 ; by taking care of (6.16) we infer from (6.19):

$$
\begin{aligned}
\sigma & \leq \eta\left\langle A_{1} \widetilde{h}, \widetilde{h}\right\rangle+|b| \int \frac{1}{r^{2}}\left(\left|h_{r}\right|^{2}+\left|h_{\varphi}\right|^{2}\right) r d r \\
& \leq-\eta c_{1}\|\widetilde{h}\|^{2}+\frac{|b|}{r_{1}^{2}}\|\widetilde{h}\|^{2}, \quad \widetilde{h}=\left(h_{r}, h_{\varphi}\right) .
\end{aligned}
$$

That is, $\sigma \leq 0$ if $|b| \leq r_{1}^{2} \eta c_{1}$

Comments. It thus follows from Prop. 6.2, 6.8 that there is $\delta_{1}=\delta_{1}(\eta)$ such that: $\left(^{*}\right)$ if $|b| \leq \delta_{1}$ then the eigenvalue problem (5.6) has only eigenvalues $\sigma \leq 0$ for any choice of $\theta \in(0,2 \pi), \alpha \in \mathbb{Z},|k| \geq 1$. Under our standing assumption $\beta \equiv 0$ the proof of Thm. 1 is thus completed if we can show:

Lemma 6.2. There is $\delta_{2}=\delta_{2}(\eta)$ as follows: if $|b| \leq \delta_{2}$ and $k=0$ then for any $\theta \in(0,2 \pi), \alpha \in \mathbb{Z}$ the associated eigenvalue problem (5.6) has only eigenvalues $\sigma \leq 0$.

Once Lemma 6.2 is proved we can combine Prop. 6.1, Lemmas 6.1, 6.1', 6.2 so as to infer

Corollary. Theorem 1 holds under the assumption $\beta=0$.

The proof of Lemma 6.2 is rather technical and relegated to an appendix. In what follows we take Lemma 6.2 for granted and drop the assumption $\beta=0$, that 
is we proceed to

Step 3: the case $\beta \neq 0$. In order to handle this case we recall the real quadratic form $J(w)$ given by (6.2). This form depends on the four parameters $\nu, \eta, b, \beta$; in order to emphasize this we write more explicitely $J(w)=J(\nu, \eta, b, \beta / w)$ whence

$$
J(\nu, \eta, b, \beta / w)=J\left(\frac{\nu}{2}, \frac{\eta}{2}, b, 0 / w\right)+J\left(\frac{\nu}{2}, \frac{\eta}{2}, 0, \beta / w\right) .
$$

To the first term on the righthand side of (6.20) we apply the corollary to Lemma 6.2, ie. Thm. 1 for $\beta=0$ so as to find $\delta_{0}=\delta_{0}\left(\frac{\nu}{2}, \frac{\nu}{2}\right)$ such that $|b| \leq \delta_{0}$ implies $J\left(\frac{\nu}{2}, \frac{\eta}{2}, b, 0 / w\right) \leq 0$ for $w \in \operatorname{dom}(\mathcal{A})$. Theorem 1 is thus proved in full if we can show that given $\nu, \eta>0$ there is $\delta_{1}=\delta_{1}(\nu, \eta)$ such that $J(\nu, \eta, 0, \beta / w) \leq 0$ if $|\beta| \leq \delta_{1}$. To prove this we set for $w=(v, h)$ :

$$
\begin{aligned}
& J^{\prime}(w)=J^{\prime}(v, h)=\int \frac{1}{r}\left(h_{r} v_{z}-h_{z} v_{r}\right) d \mu, \quad d \mu=r d r d \varphi d z \\
& J^{\prime \prime}(v)=\int \frac{1}{r}\left(\left|v_{r}\right|^{2}+\left|v_{z}\right|^{2}\right) d \mu
\end{aligned}
$$

whence

$$
J(\nu, \eta, 0, \beta / w)=\left\langle A_{S} v, v\right\rangle+\left\langle A_{S}^{\prime} h, h\right\rangle+\beta J^{\prime}(v, h) .
$$

We also observe the inequality

$$
J^{\prime}(v, h) \leq \frac{1}{2} J^{\prime \prime}(v)+\frac{1}{2} J^{\prime \prime}(h),
$$

which combined with (6.21) yields

$$
J(\nu, \eta, 0, \beta / w) \leq\left\langle A_{S} v, v\right\rangle+\frac{\beta}{2} J^{\prime \prime}(v)+\left\langle A_{S}^{\prime} h, h\right\rangle+\frac{\beta}{2} J^{\prime \prime}(h) .
$$

We note

Proposition 6.9. Given $\nu>0$ there is $\delta_{0}=\delta_{0}(\nu)$ such that $|\beta| \leq \delta_{0}$ implies

$$
\left\langle A_{S} v, v\right\rangle+\frac{\beta}{2} J^{\prime \prime}(v) \leq 0, \quad v \in \operatorname{dom}\left(A_{S}\right) .
$$

Proof. Essentially the same as that of Prop. 6.1

Our task, ie. the full proof of Thm. 1 is thus accomplished if we can show:

Lemma 6.3. Given $\eta$ there is $\delta_{1}(\eta)>0$ such that $|\beta| \leq \delta_{1}$ implies:

$$
\left\langle A_{S}^{\prime} h, h\right\rangle+\beta J^{\prime \prime}(h) \leq 0, \quad h \in \operatorname{dom}\left(A_{S}^{\prime}\right) .
$$

The proof is similar to that of Lemma 6.1 , ie. via Thms. 4, 5. We define a $3 \times 3$ matrix $M$ via

$$
\begin{aligned}
& M=M(\beta, r)=\left(m_{j k}\right), m_{11}=m_{33}=\frac{\beta}{r}, \\
& m_{j k}=0 \text { otherwise; }
\end{aligned}
$$

we identify henceforth the matrix $M$ in Thm. 5 with $M$ in (6.24); the bounded operator induced by $M$ via (5.1) is also denoted by $M$. With $M$ given by (6.24) 
the lefthand side of $(6.23)$ coincides with $\left\langle\left(A_{S}^{\prime}+\mathcal{P} M\right) h, h\right\rangle$; we are thus again in the position to apply Thm. 4 in order to reduce Lemma 6.3 to the equivalent statement

Lemma $6.3^{\prime}$. Given $\eta>0$ there is $\delta_{1}=\delta_{1}(\eta)>0$ such that $|\beta| \leq \delta_{1}$ implies: for any $k, \alpha \in \mathbb{Z}, \theta \in(0,2 \pi)$ the eigenvalue problem (5.6) (with $M$ as in (6.24) admits only eigenvalues $\sigma \leq 0$

Thus, once Lemma $6.3^{\prime}$ is proved, Thm. 1 follows. The proof of Lemma $6.3^{\prime}$ now leads again to a distinction of cases according to whether $|k| \geq 2,|k|=1$ or $k=0$.

These are handled by the following propositions.

Proposition 6.10. Let $|\beta| \leq \eta r_{2}^{-1}$, if $|k| \geq 2$ then the eigenvalue problem (5.6) (with $M$ as in (6.24)) admits only eigenvalues $\sigma \leq 0$ for any $\theta \in(0,2 \pi), \alpha \in \mathbb{Z}$.

Proof. Consider the quadratic form

$$
Q_{k}(x, y)=k^{2}|x|^{2}+k^{2}|y|^{2}+2 i k y x^{*}-2 i k x y^{*}, \quad k \in \mathbb{Z}
$$

and observe that $Q_{k} \geq 0$ if $|k| \geq 2$. Now let $h=\left(h_{r}, h_{\varphi}, h_{z}\right), p$ be a solution $\neq 0$ of the eigenvalue problem (5.21) for some $|k| \geq 2$; let $\|h\|=1$. We multiply (5.6) scalarly with $h$, taking care of the divergence condition and the form of $M$ via (6.24). As a result we obtain

$$
\begin{aligned}
\sigma= & -\eta \int\left(\left|\partial h_{r}\right|^{2}+\left|\partial^{*} h_{\varphi}\right|^{2}+\left|\partial h_{z}\right|^{2} d \mu-\eta \int \frac{1}{r^{2}} Q_{k}\left(h_{r}, h_{\varphi}\right) d \mu\right. \\
& -\eta \widehat{\alpha}^{2}-\eta \int \frac{\left|h_{r}\right|^{2}}{r^{2}} d \mu-\eta k^{2} \int \frac{\left|h_{z}\right|^{2}}{r^{2}} d \mu+\beta \int \frac{1}{r}\left(\left|h_{r}\right|^{2}+\left|h_{z}\right|^{2}\right) d \mu
\end{aligned}
$$

where $d \mu=r d r$. Since $Q_{k} \geq 0$ in view of $|k| \geq 2$, the righthand side of (6.25) is $\leq 0$ if

$$
|\beta| \int \frac{1}{r}\left(\left|h_{r}\right|^{2}+\left|h_{z}\right|^{2}\right) d \mu \leq \eta \int \frac{1}{r^{2}}\left(\left|h_{r}\right|^{2}+\left|h_{z}\right|^{2}\right) d \mu
$$

Since $\int=\int_{r_{1}}^{r_{2}}$ the claim follows from (6.26)

The next proposition takes care of the case $|k|=1$.

Proposition 6.11. There exists $\delta_{1}=\delta_{1}(\eta)>0$ such that $|\beta| \leq \delta_{1}$ implies: if $|k|=1$ then the eigenvalue problem (5.6) (with $M$ as in (6.24)) admits only eigenvalues $\sigma \leq 0$ for any $\theta \in(0, \pi), \alpha \in \mathbb{Z}$.

Proof. With $|k|=1$ and $h=\left(h_{r}, h_{\varphi}, h_{z}\right), p$ a solution $\neq 0$ of (5.6) for some $\theta \in(0,2 \pi), \alpha \in \mathbb{Z}$ and such that $\|h\|=1$, we proceed as in the proof of Prop. 6.10 so as to arrive at expression (6.25) for $\sigma$. We recall the operator $A_{\lambda}$ in (6.6) and 
set $\lambda=1$; we now rearrange terms in (6.25) so as to obtain:

$$
\begin{aligned}
\sigma & =\eta\left\langle A_{1}, \widetilde{h}, \widetilde{h}\right\rangle-\eta \int\left|\partial h_{z}\right|^{2} d \mu-\eta \widehat{\alpha}^{2}-\eta \int \frac{\left|h_{z}\right|^{2}}{r^{2}} d \mu \\
& +\beta \int \frac{\left|h_{r}\right|^{2}}{r} d \mu+\beta \int \frac{\left|h_{z}\right|^{2}}{r} d \mu, \quad \widetilde{h}=\left(h_{r}, h_{\varphi}\right) .
\end{aligned}
$$

With $c_{1}=\min (c(1), c(-1))$ we infer from Prop. 6.7 and (6.27):

$$
\sigma \leq-\eta c_{1}\|\widetilde{h}\|^{2}+\frac{|\beta|}{r_{1}}\|\widetilde{h}\|^{2}-\eta \int \frac{\left|h_{z}\right|^{2}}{r^{2}} d \mu+|\beta| r_{2} \int \frac{\left|h_{z}\right|^{2}}{r^{2}} d \mu .
$$

We thus may set $\delta_{1}=\min \left(\eta r_{1} c_{1}, \eta r_{2}^{-1}\right)$

It remains to settle the case $k=0$. To this end we note the auxiliary

Proposition 6.12. There is $c_{0}$ as follows: (*) if $f \in H^{1}(I)$ and $\int_{r_{1}}^{r_{2}} f r d r=0$ then

$$
c_{0} \int_{r_{1}}^{r_{2}}|f|^{2} r d r \leq \int_{r_{1}}^{r_{2}}|\partial f|^{2} r d r
$$

Hint of proof. It suffices to consider real $f \in C^{1}(I)$. If $\int_{r_{1}}^{r_{2}} f r d r=0$ then $f\left(r_{0}\right)=0$ for some $r_{0} \in\left(r_{1}, r_{2}\right)$. From this observation the claim follows along standard lines first for $f \in C^{1}(I)$ and then by approximation for $f \in H^{1}(I)$

The last step in the proof of Thm 1 is now provided by

Proposition 6.13. There is $\delta_{1}=\delta_{1}(\eta)>0$ such that $|\beta| \leq \delta_{1}$ implies: if $k=0$ then the eigenvalue problem (5.6) (with $M$ as in (6.24)) admits only eigenvalues $\sigma \leq 0$ for any $\theta \in(0,2 \pi), \alpha \in \mathbb{Z}$.

Proof. Let $h=\left(h_{r}, h_{\varphi}, h_{z}\right), p$ be a solution $\neq 0$ of (5.6)(i)-(iv) for some $\theta \in(0,2 \pi)$ but with $k=0$; let $\|h\|=1$. Since $\theta \in(0,2 \pi)$ we have $\widehat{\alpha} \neq 0[(5.3)]$, whence $\int_{r_{1}}^{r_{2}} h_{z} r d r=0$ by virtue of $(5.6)(\mathrm{iv})$ and since $h_{r} \in H^{2}(I) \cap H_{0}^{1}(I)$. That is (6.28) holds for $f=h_{z}$. We now multiply (5.6)(i)-(iii) scalarly with $h$; by taking care of (5.6)(iv) we get

$$
\begin{aligned}
\sigma & =-\eta \int\left(\left|\partial h_{r}\right|^{2}+\left|\partial^{*} h_{\varphi}\right|^{2}\right) d \mu-\widehat{\alpha}^{2} \eta-\int \frac{\left|h_{r}\right|^{2}}{r^{2}} d \mu-\eta \int\left|\partial h_{z}\right|^{2} d \mu \\
& +\beta \int \frac{\left|h_{r}\right|^{2}}{r} d \mu+\beta \int \frac{\left|h_{z}\right|^{2}}{r} d \mu \\
& \leq-\eta \int \frac{\left|h_{r}\right|^{2}}{r^{2}} d \mu-\eta \int\left|\partial h_{z}\right|^{2} d \mu+|\beta| \int \frac{\left|h_{r}\right|^{2}}{r} d \mu+|\beta| \int \frac{\left|h_{z}\right|^{2}}{r} d \mu
\end{aligned}
$$

where $d \mu=r d r, \int=\int_{r_{1}}^{r_{2}}$. The existence of $\delta_{1}(\eta)$ now follows from this inequality and (6.28)

Addendum. Proposition 3.6, which claims $A_{S}^{\prime} \leq 0$, now follows obviously as special case from Lemma 6.1 for $b=0$. How to prove Prop. 3.6 without detour 
via Bloch space theory is not known to us.

\section{Open problems}

An inspection of the proof of Thm. 1 shows that weak energy stability does not depend on the value of $\alpha$. It is in the proof of Thm. 2 where $\alpha$ is required to be small (see [16] for the same situation in the MBP). This suggests the problem: if $\beta=0$, does one find $\delta=\delta(\eta, \nu)$ such that $|a|,|b| \leq \delta$ implies Ljapounov stability for any value $\alpha$ ? Another question is whether one can improve Thm. 3 by proving asymptotic stability. One option is to extend the method of diffusive stability, successfully applied to Couette-Taylor vortices in [21], to the present case. Whether this can be done remains open.

\section{Appendix: Proof of Lemma 6.2}

The proof is an essentially straightforward application of the perturbation theory of a simple eigenvalue. For reasons of space we have to content us with a condensed presentation. Routine computations are omitted whenever possible. By Lemma 6.2 we have to study the system (5.6), (5.7) for the case where $\alpha \in \mathbb{Z}, \theta \in[0,2 \pi]$ and hence $\widehat{\alpha}$ are arbitrary, but where $k=0$; the matrix $M$ is thereby given by (6.4). The first step amounts to study for $\widehat{\alpha}$ small, that is for $\widehat{\alpha} \neq 0$ and small and for $\widehat{\alpha}=0$. The case $\widehat{\alpha} \neq 0$ is based on (5.6)(i)-(iv), while the case $\widehat{\alpha}=0$ is based on $(5.6),(5,7)(\mathrm{i}),(\mathrm{ii}),\left(\right.$ (iii) $^{\prime}$, (iv). Now (iii) ${ }^{\prime}$ contains an auxiliary constant $c$ small, which however must be $=0$ in view of $\widehat{\alpha}=0$, the assumption $h_{3} \in \widetilde{H}^{2}(I)$ and the condition $\int h_{3} r d r=0$ in (iii) $)^{\prime}$. Thus we are left with the study of (5.6)(i)-(iv) for $k=0$ and $\widehat{\alpha}$ small. Thus let $h_{1}=h_{r}, h_{2}=h_{\varphi}, h_{3}=h_{z}, p$ be a solution of the eigenvalue problem (5.6)(i)-(iv) for some $\sigma$, with $k=0$. We multiply equation (i) with $i \widehat{\alpha}$, apply $\partial$ to (iii) and subtract the second result from the first. As a result we obtain

$$
\eta\left(\partial \partial^{*}-\widehat{\alpha}^{2}\right)\left(i \widehat{\alpha} h_{r}-\partial h_{z}\right)-\frac{i \widehat{\alpha} b h_{\varphi}}{r^{2}}=\sigma\left(i \widehat{\alpha} h_{r}-\partial h_{z}\right)
$$

We now use (iv) and replace $-i \widehat{\alpha} \partial h_{z}$ by $\partial \partial^{*} h_{r}$. Together with (ii) we are then left with a couple of equations not containing $h_{z}, p$ :

$$
\begin{aligned}
\eta\left(\partial \partial^{*}-\widehat{\alpha}^{2}\right)\left(\partial \partial^{*}-\widehat{\alpha}^{2}\right) h_{r}+\frac{\widehat{\alpha}^{2} b h_{\varphi}}{r^{2}} & =\sigma\left(\partial \partial^{*}-\widehat{\alpha}^{2}\right) h_{r} \\
\eta\left(\partial \partial^{*}-\widehat{\alpha}^{2}\right) h_{\varphi}-\frac{b h_{r}}{r^{2}} & =\sigma h_{\varphi}
\end{aligned}
$$

From (iv) and since $h_{z} \in \widetilde{H}^{2}(I)$ we have a further boundary condition for $h_{r}$ :

$$
\partial \partial^{*} h_{r}=0 \quad \text { on } \quad \partial I, \quad \text { ie. } \quad \partial \partial^{*} h_{r} \in H^{2}(I) \cap H_{0}^{1}(I) .
$$


In order to rewrite (a.2) abstractly we stipulate

$$
\begin{aligned}
& B=\partial \partial^{*}, \operatorname{dom}(B)=H^{2}(I) \cap H_{0}^{1}(I), \quad A=B^{2}, \\
& C=\partial \partial^{*}, \operatorname{dom}(C)=\widehat{H}^{2}(I) ; \quad T=\operatorname{diag}(A, C) .
\end{aligned}
$$

We furthermore set:

$$
\begin{aligned}
B(\widehat{\alpha}, \sigma, b) & =\left(b_{j k}\right) \text { with } b_{11}=\left(2 \widehat{\alpha}^{2} \eta+\sigma\right) B-\widehat{\alpha}^{2} \sigma-\eta \widehat{\alpha}^{4} \\
b_{12} & =-\frac{\widehat{\alpha}^{2} b}{r^{2}}, \quad b_{21}=0, \quad b_{22}=\eta \widehat{\alpha}^{2}+\sigma \\
G & =\left(g_{j k}\right) \text { with } g_{21}=\frac{1}{r^{2}}, \quad g_{11}=g_{22}=g_{12}=0 .
\end{aligned}
$$

Based on (a.4), (a.5) and some rearrangements of terms we can rewrite (a.2) abstractly as follows:

$$
(\eta T-b G) h=B(\widehat{\alpha}, \sigma, b) h, \quad h=\left(h_{r}, h_{\varphi}\right) \in \operatorname{dom}(A) \times \operatorname{dom}(C) .
$$

We observe that $T$ is selfadjoint in $\mathcal{L}^{2}(I)^{2}$, while $B(\widehat{\alpha}, \sigma, b)$ is bounded relative to $T$ and thus relative to $\eta T-b G$ with relative bound zero. It can easily be shown that the original problem, ie. (5.6)(i)-(iv) for $k=0$ is fully equivalent to (a.6). In order to apply perturbation theory to (a.6) we consider $B(\widehat{\alpha}, \sigma, b)$ as a small relatively bounded perturbation of $\eta T-b G$. In order to study the kernel of $\eta T-b G$ let $e=\left(e_{1}, e_{2}\right) \in \operatorname{dom}(T)$ satisfy $(\eta T-b G) e=0$. By (a.4), (a.5) this leads to

$$
\begin{aligned}
& A e_{1}=0, \quad C e_{2}=0 \text { whence } e_{1}=0, e_{2}=\frac{\zeta}{r}, \text { ie. up to } \\
& \text { a factor, } e=\left(0, \frac{1}{r}\right) .
\end{aligned}
$$

To determine the algebraic multiplicity of $0 \in \sigma(\eta T-b G)$, let $f=\left(f_{1}, f_{2}\right)$ satisfy

$$
(\eta T-b G) f=e .
$$

By (a.4), (a.5), (a.7), equation (a.8) decomposes into $A f_{1}=0, \eta C f_{2}=\frac{1}{r}$ whence

$$
\eta\left(C f_{2}, \frac{1}{r}\right)=\left(\frac{1}{r}, \frac{1}{r}\right) \text {, ie. } \eta\left(f, C \frac{1}{r}\right)=\left(\frac{1}{r}, \frac{1}{r}\right)>0 .
$$

Since $C \frac{1}{r}=0$, a contradiction arises from (a.9), that is, (a.8) has no solution; therefore the algebraic multiplicity of $0 \in \sigma(\eta T-b G)$ is $=1$. Here and below, $($,$) is the scalar product in \mathcal{L}^{2}(I)$ with measure $d \mu=r d r$, while $\langle$,$\rangle denotes the$ scalar product induced by $($,$) on \mathcal{L}^{2}(I)^{2}$. It follows that $\sigma=0$ is an eigenvalue of algebraic multiplicity $=1$ of the adjoint

$$
(\eta T-b G)^{*}=\eta T-b G^{t}, \quad G^{t}=\text { transpose of } G .
$$

The kernel of the adjoint is spanned by an element $e_{+}=\left(e_{1}^{+}, e_{2}^{+}\right)$such that

$$
C e_{2}^{+}=0, \quad \eta A e_{1}^{+}=\frac{b}{r^{2}} e_{2}^{+} .
$$

That is we have up to a scalar multiple:

$$
e_{+}=\left(b \phi_{0}, \frac{1}{r}\right), \quad \phi_{0}=(\eta A)^{-1} \frac{1}{r^{3}} .
$$

The projection $K_{b}$ which projects onto $\operatorname{rg}(\eta T-b G)$ is now given by

$$
K f=K_{b} f=f-\left\langle e, e_{+}\right\rangle^{-1}\left\langle f, e_{+}\right\rangle e .
$$


Based on this notions we can apply standard perturbation theory to (a.6). This amounts to seek a solution pair $h=h(\widehat{\alpha}, b), \sigma=\sigma(\widehat{\alpha}, b)$ for small $\widehat{\alpha}, b$ with $h$ of the form

$$
h=e+g(\widehat{\alpha}, b), \quad\left\langle g(\widehat{\alpha}, b), e_{+}\right\rangle=0, \text { ie. } K_{b} g=g .
$$

In a first step we replace $h$ by $e+g$ in (a.6) with $K g=g$ assumed. To the resulting equation we apply $K=K_{b}$ and note that $K$ commutes with $\eta T-b G$. Since $K g=g$, we obtain as a result

$$
K(\eta T-b G) g=K B(\widehat{\alpha}, \sigma, b)(e+g), \quad K g=g .
$$

This equation is solved for $g$; by a standard computation we obtain

$$
\begin{aligned}
& g(\widehat{\alpha}, \sigma, b)=\left\{1-H^{-1} K B(\widehat{\alpha}, \sigma, b)\right\}^{-1} H^{-1} K B(\widehat{\alpha}, \sigma, b), \\
& H=K(\eta T-b G) .
\end{aligned}
$$

Here, implicit use is made of the fact that $\eta T-b G$ is boundedly invertible on its invariant subspace $\operatorname{rg}(K)=\operatorname{rg}(\eta T-b G)$. As to $g(\widehat{\alpha}, \sigma, b)$, the following proposition is proved straightforwardly:

Proposition a.1. Given $N$ there is $\delta_{0}$ as follows: $g(\widehat{\alpha}, \sigma, b)$ is analytic for $|b| \leq N$, $|\widehat{\alpha}|,|\sigma| \leq \delta_{0}$ with values in the Banach space

$$
X=\operatorname{rg}(\eta T-b G) \cap \operatorname{dom}(T), \quad\|f\|_{X}=\|T f\|+\|f\| .
$$

Now $g=g(\widehat{\alpha}, \sigma, b)$ thus found is a solution of (a.12); since $K$ commutes with $\eta T-b G$ it cancels on the lefthand side of (a.12). It follows that $h=e+g$ is a solution of (a.6) if and only if the following orthogonality condition holds:

$$
\left\langle\mathcal{B}(\widehat{\alpha}, \sigma, b)(e+g(\widehat{\alpha}, \sigma, b)), e_{+}\right\rangle=F(\widehat{\alpha}, \sigma, b)=0 .
$$

Our aim is to seek $\sigma=\sigma(\widehat{\alpha}, b)$ such that (a.14) holds. Since $F(\widehat{\alpha}, \sigma, b)$ is analytic in $\widehat{\alpha}, \sigma, b$ (Prop. a.1) this suggests to apply the implicit function theorem and to seek a solution $\sigma(\widehat{\alpha}, b)$ of the form

$$
\sigma(\widehat{\alpha}, b)=\sigma_{0}(b)+\widehat{\alpha} \sigma_{1}(b)+\widehat{\alpha}^{2} \sigma_{2}(b) \ldots, \widehat{\alpha}, b \text { small }
$$

for the solution $\sigma$ of (a.14). In order to determine the coefficients $\sigma_{j}(b)$ by a comparison of coefficients we recall $\mathcal{B}(\widehat{\alpha}, \sigma, b)$ in (a.5) which has the form:

$$
\begin{aligned}
& \mathcal{B}(\widehat{\alpha}, \sigma, b)=B_{10} \sigma+B_{02} \widehat{\alpha}^{2}+\mathcal{B}_{12} \sigma \widehat{\alpha}^{2}+\mathcal{B}_{04} \widehat{\alpha}^{4}, \\
& \mathcal{B}_{10}=\left(\begin{array}{ll}
B & 0 \\
0 & 1
\end{array}\right), \quad \mathcal{B}_{02}=\left(\begin{array}{rr}
2 \eta B & -\frac{b}{r^{2}} \\
0 & \eta
\end{array}\right), \quad \mathcal{B}_{12}=\left(\begin{array}{rr}
-1 & 0 \\
0 & 0
\end{array}\right) \\
& \mathcal{B}_{04}=\left(\begin{array}{rr}
-\eta & 0 \\
0 & 0
\end{array}\right), \quad \mathcal{B} \text { via }(a .4)
\end{aligned}
$$

Moreover since $\mathcal{B}(0,0, b)=0$ we infer from (a.13), (a.16):

$$
g(\widehat{\alpha}, \sigma, b)=g_{10}(b) \sigma+g_{01}(b) \widehat{\alpha}+\text { higher order terms. }
$$


Inserting (a.16), (a.17) into (a.14) we see that $F(\widehat{\alpha}, \sigma, b)$ has the form:

$$
\begin{aligned}
& F(\widehat{\alpha}, \sigma, b)=\sigma\left\langle\mathcal{B}_{10} e, e_{+}\right\rangle+\widehat{\alpha}^{2}\left\langle\mathcal{B}_{02} e, e_{+}\right\rangle+\widehat{\alpha} \sigma f_{11}(b)+ \\
& =f_{20}(b) \sigma^{2}+\text { higher order terms, }
\end{aligned}
$$

whereby

$$
\begin{aligned}
& \left\langle\mathcal{B}_{10} e, e_{+}\right\rangle=\left(\frac{1}{r}, \frac{1}{r}\right), \quad\left\langle\mathcal{B}_{02} e, e_{+}\right\rangle=-b^{2}\left((\eta A)^{-1} \frac{1}{r^{3}}, \frac{1}{r^{3}}\right)+\eta\left(\frac{1}{r}, \frac{1}{r}\right) \\
& \text { with }\left(\frac{1}{r}, \frac{1}{r}\right)>0, \quad\left((\eta A)^{-1} \frac{1}{r^{3}}, \frac{1}{r^{3}}\right)>0 .
\end{aligned}
$$

This enables us to apply the implicit function theorem so as to conclude:

Proposition a.2. There are $\alpha_{0}, \sigma_{0}, b_{0}>0$ and a complex holomorphic function $\sigma(\widehat{\alpha}, b),|\widehat{\alpha}| \leq \alpha_{0},|b| \leq b_{0}$ such that:

(a) $F(\widehat{\alpha}, \sigma(\widehat{\alpha}, b), b)=0$ for $|\widehat{\alpha}| \leq \alpha_{0},|b| \leq b_{0}$, (b) $\sigma(0, b)=0$,

(c) if $F(\widehat{\alpha}, \sigma, b)=0$ and $|\widehat{\alpha}| \leq \alpha_{0},|\sigma| \leq \sigma_{0},|b| \leq b_{0}$

then $\sigma=\sigma(\widehat{\alpha}, b)$.

By Prop. a.2, $\sigma(\widehat{\alpha}, b)$ admits a series expansion of type (a.15) with coefficients $\sigma_{j}(b)$ holomorphic in $|b| \leq b_{0}$; in particular $\sigma_{0}(b)=0$ by (b) of Prop. a.2. In order to determine $\sigma_{1}(b), \sigma_{2}(b)$ we insert the expansion (a.15) into (a.18) and perform a comparison of coefficients. We find

$$
\sigma_{2}(b)=\left(\frac{1}{r}, \frac{1}{r}\right)^{-1}\left(b^{2}\left((\eta A)^{-1} \frac{1}{r^{3}}, \frac{1}{r^{3}}\right)-\eta\left(\frac{1}{r}, \frac{1}{r}\right)\right), \sigma_{1}(b)=0 .
$$

Based on (a.20) it follows that $\sigma(\widehat{\alpha}, b)$ has a representation:

$$
\begin{gathered}
\sigma(\widehat{\alpha}, b)=\left(\frac{1}{r}, \frac{1}{r}\right)^{-1}\left(b^{2}\left((\eta A)^{-1} \frac{1}{r^{3}}, \frac{1}{r^{3}}\right)-\eta\left(\frac{1}{r}, \frac{1}{r}\right)\right) \widehat{\alpha}^{2}+\widehat{\alpha}^{3} G(\widehat{\alpha}, b), \\
G(\widehat{\alpha}, b) \text { holomorphic in }|\widehat{\alpha}| \leq \alpha_{0},|b| \leq b_{0} .
\end{gathered}
$$

With $G(\widehat{\alpha}, b)$ holomorphic, there is $M>0$ with $|G(\widehat{\alpha}, b)| \leq M$ for $|\widehat{\alpha}| \leq \alpha_{0}$, $|b| \leq b_{0}$. By this remark and (a.21) we conclude

Proposition a.3. There are $\alpha_{1} \in\left(0, \alpha_{0}\right], b_{1} \in\left(0, b_{0}\right]$ such $|\widehat{\alpha}| \leq \alpha_{1},|b| \leq b_{1}$, $\widehat{\alpha}, b \in \mathbb{R}$ imply

$$
\sigma(\widehat{\alpha}, b) \leq-\frac{\eta}{2} \widehat{\alpha}^{2}
$$

Prop. a. 3 constitutes the major step in the proof of Lemma 6.2; however, two supplements are needed. As to the first, let $\widehat{\alpha}=0$. Since $k=0$, the divergence condition $(5.6)(\mathrm{iv})$ reduces to $\partial^{*} h_{r}=0$ whence $h_{r}=0$ since $h\left(r_{j}\right)=0$ at $r_{j}$, $j=1,2$. The system (a.2) or equivalently (a.6) now reduces to the Robin type boundary value problem $\eta \partial \partial^{*} h_{\varphi}=\sigma h_{\varphi}, \partial\left(r h_{\varphi}\right)=0$ at $r_{1}, r_{2}$. This system has a $b$-independent spectrum $\sigma_{0}=0>\sigma_{1}>\ldots$ A slight perturbation $\widehat{\alpha}, b$ of $\widehat{\alpha}=0$, $b=0$ such that $|\widehat{\alpha}| \leq \alpha_{1},|b| \leq b_{1}$ induces a slight perturbation of the spectrum $\sigma_{j}, j \geq 0$. The perturbation of $\sigma(\widehat{\alpha}, b)$ of $\sigma_{0}=0$ in particular behaves according Prop. a.3. 
In order to describe the behaviour of the other eigenvalues we recall $B, C$ in (a.4) and multiply (a.2) with $\left(B-\widehat{\alpha}^{2}\right)^{-1}$ so as to obtain the system

$$
\begin{aligned}
\eta B h_{r}-\eta \widehat{\alpha}^{2} h_{r}+\widehat{\alpha}^{2} b\left(B-\widehat{\alpha}^{2}\right)^{-1} \frac{h_{\varphi}}{r^{2}} & =\sigma h_{r} ; \quad h_{r} \in \operatorname{dom}(B), \\
\eta C h_{\varphi}-\eta \widehat{\alpha}^{2} h_{\varphi}-\frac{b h_{r}}{r^{2}} & =\sigma h_{\varphi} ; \quad h_{p} \in \operatorname{dom}(C) .
\end{aligned}
$$

(a.23) is an eigenvalue problem in standard form, which is equivalent to (a.2) resp. (a.6). In order to apply Thm. 3.16 in [9], IV. $\S 4$ we fix $d \in\left(\sigma_{1}, 0\right)$ arbitrarily. With $\alpha_{1}, b_{1}$ as in Prop. a.3 we then have

Proposition a.4. There are $\alpha_{2} \in\left(0, \alpha_{1}\right], b_{2} \in\left(0, b_{1}\right]$ such that $|\widehat{\alpha}| \leq \alpha_{2},|b| \leq b_{2}$ implies: if $\sigma$ is an eigenvalue of (a.23) (or equivalently of (a.2), (a.6)) then either $\sigma<d$ or else $\sigma=\sigma(\widehat{\alpha}, b)$.

Propositions a.3, a.4 together prove Lemma 6.2 under the proviso that $\widehat{\alpha}, b$ are both small. In order to complete the proof we have to get rid of the smallness condition for $\widehat{\alpha}$. This is accomplished by

Proposition a.5. Let $c>0$ and assume $|\widehat{\alpha}| \geq c,|b| \leq c^{2} r_{1}^{2} \eta$. If $\sigma$ is an eigenvalue of (a.2) (or equivalently of (5.6)(i)-(iv), $k=0$ ) then $\sigma \leq 0$.

Proof. We take (5.6)(i)-(iv), $k=0$ as starting point and assume that a solution $h=\left(h_{r}, h_{\varphi}, h_{z}\right) \neq 0, p$ is given for some $\widehat{\alpha}, b$ subject to the assumptions; we may assume that $\|h\|=1$. We now multiply (5.6)(i)-(iii) scalarly with $h$ and take $\|h\|=1$ into account; we then obtain

$$
\begin{aligned}
\sigma= & -\int\left(\left|\partial^{*} h_{r}\right|^{2}+\left|\partial^{*} h_{\varphi}\right|^{2}+\left|\partial h_{z}\right|^{2}\right) r d r-\widehat{\alpha}^{2} \eta \\
& -b \int \frac{1}{r^{2}}\left(h_{\varphi} h_{r}^{*}+h_{r} h_{\varphi}^{*}\right) r d r
\end{aligned}
$$

From our assumptions and (a.24) we obtain after a minor computation the inequality

$$
\sigma \leq-\eta c^{2}+\frac{|b|}{r_{1}^{2}} \int\left(\left|h_{r}\right|^{2}+\left|h_{\varphi}\right|^{2}+\left|h_{z}\right|^{2}\right) r d r \leq-\eta c^{2}+\frac{|b|}{r_{1}^{2}}
$$

The claim now follows from (a.25) and our assumptions

The final step in the proof of Lemma 6.2 now follows by combining Prop. a.3-a.5.

Proposition a.6. Let $|b| \leq \min \left(b_{2}, \eta \alpha_{2}^{2} r_{1}^{2}\right)$; if $\sigma$ is an eigenvalue of (5.6)(i)-(iv) with $k=0$ (or equivalently of (a.2)) then $\sigma \leq 0$.

Proof. Case 1: $|\widehat{\alpha}| \leq a_{2}$. Since $|b| \leq b_{2}$ we can apply Prop. a.4 and infer that either $\sigma<d$ or else $\sigma=\sigma(\widehat{\alpha}, b)$. Since $d<0$ we have also $\sigma \leq 0$ in the first case. As to the second case we note that $\alpha_{2} \leq \alpha_{1}, b_{2} \leq b_{1}$ what allows us to apply Prop. a. 3 so as to conclude $\sigma=\sigma(\widehat{\alpha}, b) \leq 0$. 
Case 2. $|\widehat{\alpha}| \geq \alpha_{2}$. Since $|b| \leq \eta \alpha_{2}^{2} r_{1}^{2}$ we are in the situation of Prop. a.5 which allows us to infer $\sigma<0$

Remark. Lemma 6.2 follows from Prop. a. 6 by setting $\delta_{2}=\delta_{2}(\eta)=\min \left(b_{2}, \eta \alpha_{2}^{2} r_{1}^{2}\right)$.

\section{References}

[1] H. Amann, Linear and quasilinear parabolic problems I. Birkhäuser, Basel, Boston 1995.

[2] S. Chandrasekhar, Hydrodynamic and hydromagnetic stability. Dover Publ. New York 1961.

[3] P. Chossat, G. Iooss, The Couette-Taylor problem. Springer, New York, Berlin 1991.

[4] P. Drazin, W. Reid, Hydrodynamic stability. Cambridge University Press, London, New York 1981.

[5] A. Friedman, Partial Differential Equations. R. E. Krieger Publishing Comp. Inc. Malabor, FL 1969.

[6] G. P. Galdi, M. Padula, A new approach to energy theory in the stability of fluid motion. Arch. Rat. Mech. Anal. 110 (1990), 187-296.

[7] A. Georgescu, Hydrodynamic stability. Martinus Nijhoff Publ. Amsterdam 1985.

[8] D. Gilbarg, D. Trudinger, Elliptic partial differential equations of second order. Springer, Berlin 1981.

[9] D. Henningson, J. Schmid, Stability and transitions in shear flows. Springer, New York 2001.

[10] T. Kato, Perturbation theory for linear operators. Springer, Berlin 1991.

[11] R. Meyer-Spasche, Pattern formation in viscous flows. Birkhäuser, Boston, Basel 1999.

[12] A. Mielke, Instability and stability of rolls in the Swift-Hohenberg equations. Commun. Math. Phys. 189 (1997), 829-853.

[13] A. Pazy, Semigroups of linear operators and applications to PDE's. Appl. Math. Sci. Vol. 44, Springer, New York 1983.

[14] H. Ponce, R. Racke, T. Sideris, E. Titi, Global stability of large solutions to 3D Navier-Stokes equations. Comm. Math. Physics 159 (1984), 329-341.

[15] B. Scarpellini, Loss of stability in the magnetic Bénard problem. NoDEA 7 (2000), 157-185.

[16] B. Scarpellini, Large solutions of the magnetic Bénard problem on the infinite layer. Nonlinear Analysis 48 (2002), 85-116.

[17] B. Scarpellini, Spatially decaying perturbations of periodic equilibria of Couette-Taylor. Manuscript.

[18] B. Scarpellini, On properties of some Stokes-operators of Couette-Taylor. C. R. Acad. Sci. Paris, t. 331, Série 1, p. 673-946, 2000.

[19] B. Scarpellini, Stability, instability and direct integrals. Chapman \& Hall/CRC, Boca Raton, Fl. 1999.

[20] B. Scarpellini, Stability properties related to the magnetic Bénard problem. Topics in Nonlinear Analysis, Birkhäuser, Basel (1999), 585-610.

[21] G. Schneider, Nonlinear stability of Taylor-vortices in infinite cylinders. Arch. Rat. Mech. Anal. 144 (1999), 121-200.

[22] H. Sohr, The Navier-Stokes equations. Birkhäuser, Basel, Boston 2001.

[23] B. Straugham, The energy method, stability and nonlinear convection. Appl. Math. Sci. 91, Springer, New York 1992.

[24] W. von Wahl, The Boussinesq-equations in terms of poloidal and toroidal fields and the mean flow. Bayreuther Math. Schriften 40 (1992) 203-29.

[25] M. Reed, B. Simon, Methods of Math. Physics IV. Academic Press, New York 1978.

[26] V. A. Romanov, Stability of plane parallel Couette flows. Functional analysis and its applications 7 (1973), 137-146. 
B. Scarpellini

Mathematisches Institut

Universität Basel

Rheinsprung 21

CH-4051 Basel

Schwitzerland

(Received: February 5, 2003; revised: September 29, 2003)

(1) To access this journal online:

(20) http://www.birkhauser.ch 